\title{
SÁNDOR JENEI@ Group Representation for Even and Odd Involutive Commutative Residuated Chains
}

\begin{abstract}
For odd and for even involutive, commutative residuated chains a representation theorem is presented in this paper by means of direct systems of abelian $o$-groups equipped with further structure. This generalizes the corresponding result of J. M. Dunnabout finite Sugihara monoids.
\end{abstract}

Keywords: Involutive residuated lattices, Construction, Representation, Abelian o-groups. Mathematics Subject Classification: Primary 97H50, 20M30; Secondary 06F05, 06F20, 03B47.

\section{Introduction}

J. M. Dunn has described the structure of finite Sugihara monoids by showing in [2] that subdirectly irreducible Sugihara monoids are linearly ordered and by describing the structure of finite Sugihara chains in [15]. We generalize Dunn's results by dropping the idempotence and the finiteness axioms.

In line with the tradition of representing classes of residuated lattices by simpler and better known structures such as groups or Boolean algebras, Mundici's celebrated categorical equivalence theorem represents MValgebras - a variety which corresponds to Łukasiewicz logic $\mathbb{L}[12]$ - by -groups with strong units using a truncation construction [43]. By dropping the divisibility axiom of MV-algebras one obtains the variety of IMTLalgebras which correspond to the logic $\mathbb{I M T L}[5,9,10,13,14,16,17,24,37,44$, 45]. The non-integral analogue of the class of IMTL-algebras shall be represented by $o$-groups in this paper. By replacing the integrality axiom of IMTL-algebras by one of its two natural non-integral analogues one obtains the class of odd and the class of even involutive semilinear $\mathrm{FL}_{e}$-algebras, the former of which is also known as the class of IUL ${ }^{f p}$-algebras corresponding

Presented by Francesco Paoli; Received August 21, 2021 
to the Involutive Uninorm Logics with fixed point $\left(\mathbb{I} \mathbb{L}^{f p}\right)$ introduced by G. Metcalfe in [38], see also [39]. We shall represent all subdirectly irreducible members of these two classes by means of abelian o-groups. Subdirectly irreducible even or odd involutive $\mathrm{FL}_{e}$-algebras are linearly ordered. Little is known about the structure of IMTL-algebras and an effective structural description for this class seems to be out of reach at present, arguably, such a structural description is impossible. However, as shown in this paper, the non-integral counterpart of the class of IMTL-algebras possesses a representation by groups, similar to MV-algebras or to cancellative commutative residuated lattices [41].

Whether Involutive Uninorm Logic $(\mathbb{I U L})$ is standard complete or not is a problem posed by G. Metcalfe and F. Montagna [39] and has become a long-standing open problem which has invoked considerable effort to solve it with no avail ${ }^{1}$. The representation theorem in this paper may serve as a step toward settling the problem of standard completeness of $\mathbb{I U L}$ in an algebraic manner.

Group theory is the science of symmetries whereas the theory of inverse semigroups is the science of partial symmetries [35]. Our main result is reminiscent to the representation theorem of a subclass of inverse semigroups, called Clifford semigroups. Every Clifford semigroup is isomorphic to a strong semilattice of groups [35, Theorem 12 in Section 5.2] (see also [51]), and the starting point of the strong semilattice construction is a direct system of groups indexed by a semilattice. In our case the starting point is a direct system of abelian $o$-groups indexed by a chain and equipped with some extra structure. The definition of the product in the strong semilattice construction is of the same Płonka sum fashion (see [48]) as in our construction in (4.8). However, in our case we need to modify the abelian o-groups prior to applying (4.8), and we also need to handle the ordering, the residual operation, the isotonicity of the product with respect to the ordering etc. In particular, we introduce in this paper an ordering on Płonka sums, called the directed lexicographic order.

Płonka sums have found applications not only in computer science, in particular in the theory of program semantics [49], but recently its corresponding regular varieties have been unexpectedly connected to logic. They

\footnotetext{
${ }^{1}$ Recently it is claimed to be proved in [52] by using proof-theoretic methods. However, the scientific community has doubts about the correctness of the proof, see the remark of the author himself in [52, second section in page 43].
} 
provide algebraic semantics to logics obtained by imposing a deductive filter to other logics; for instance, Paraconsistent Weak Kleene logic coincides with the regularization of the variety of Boolean algebras $[7,8]$.

Integral residuated lattices have been widely studied in the literature $[1,4,6,12,22,25,34]$. However, as noted by N. Galatos and J. G. Raftery in [20], non-integral residuated structures and consequently, substructural logics without the weakening rule are less understood than their integral counterparts. To overcome this, they established category equivalences to carry over the algebraic knowledge on integral structures to non-integral ones $[20,21]$. These kind of categorical approaches use some algebraic background knowledge which is available if idempotency is postulated. Our paper makes a step in the direction of providing an algebraic insight into the nonidempotent, non-integral, non-divisible setting.

Prominent examples of odd involutive $\mathrm{FL}_{e}$-algebras are abelian $\ell$-groups [3] and odd Sugihara monoids. These two classes of algebras represent two extremities: there is a single idempotent element in any lattice-ordered abelian group, whereas all elements are idempotent in any odd Sugihara monoid. The former class constitutes an algebraic semantics of Abelian Logic $[11,40,47]$ while the latter constitutes an algebraic semantics of $\mathbb{I} \mathbb{M L}^{*}$, which is a logic at the intersection of relevance logic and many-valued logic [20]. Our representation theorem puts these two rather distant logics (and more) under the same methodological umbrella.

Despite the extensive literature devoted to classes of residuated lattices, there are still few effective structural descriptions. All these results in $[1,12$, $22,23,25,33,34,36,42,46,50]$ (and also others where the focus is not a structural description in the first place such as in $[20,21]$ for example) postulate semilinearity which renders the subdirectly irreducible members linearly ordered, and either integrality together with the naturally ordered condition ${ }^{2}$ or idempotency. Our study contributes to the structural description of residuated lattices which are semilinear but neither integral nor naturally ordered nor idempotent; a first such effective structural description to the best of our knowledge besides [31,32].

A representation theorem has been presented in [31,32] for those odd involutive $\mathrm{FL}_{e}$-chains where the number of idempotent elements of the algebra is finite by means of partial sublex products of abelian o-groups, which are well understood mathematical objects that are much more regular than what had been expected to need for describing these particular $\mathrm{FL}_{e}$-chains. In the present paper we prove a representation theorem for both even and

\footnotetext{
${ }^{2}$ Or its dual notion, called divisibility.
} 
odd involutive $\mathrm{FL}_{e}$-chains without assuming any constrains on the set of their idempotent elements. The conic case is also settled. While the construction in the representation of $[31,32]$ is done by starting with an abelian $o$-group and iteratively enlarging it by other abelian o-groups until the obtained structure becomes isomorphic to the given algebra, here we present a structural description using direct systems, without referring to iteration. To this end, a core auxiliary result concerns a one-to-one correspondence between two lattice ordered classes: odd involutive $\mathrm{FL}_{e}$-algebras and even involutive $\mathrm{FL}_{e}$-algebras with an idempotent falsum constant. Here we make use of and further develop ideas of E. Casari [11], see Section 6.

Since $\ell$-groups are very specific mathematical objects compared to residuated lattices, having a representation of a class of residuated lattices by subclasses of $\ell$-groups is a rarity. It is reasonable to expect that the inherent larger complexity of the more general classes of residuated lattices renders such a representation, if exists, quite involved in its constructional part. This phenomenon can be observed in [31,32], for example, where the notions of two different partial sublex product constructions together with the technique of iteration have been used to describe the class of residuated lattices which is in our focus in the present paper with the additional assumption that the number of idempotent elements of the algebra is finite. In the present paper the complexity of the residuated structure is coded in the system of homomorphisms and in the delicate way of constructing the algebra from the direct system. Applications of the main result of this paper to amalgamation and densification problems are foreshadowed in [30].

\section{Preliminaries}

An $F L_{e}$-algebra is a structure $(X, \wedge, \vee, \cdot, \rightarrow, t, f)$ such that $(X, \wedge, \vee)$ is a lattice, $(X, \leq, \cdot, t)$ is a commutative, residuated monoid (the unit element $t$ is also referred to as the truth constant), and $f$ is an arbitrary constant, called the falsum constant. Being residuated means that there exists a binary operation $\rightarrow$, called the residual operation of $\cdot$, such that $x y \leq z$ if and only if $x \rightarrow z \geq y$. This equivalence is called adjointness condition, $(\cdot, \rightarrow)$ is called an adjoint pair. Equivalently, for any $x, z$, the set $\{v \mid x v \leq z\}$ has its greatest element, and $x \rightarrow z$, the residuum of $x$ and $z$, is defined as this element: $x \rightarrow z:=\max \{v \mid x v \leq z\}$; this is called the residuation condition. Being residuated implies that $\cdot$ is increasing. One defines the residual complement operation by $x^{\prime}=x \rightarrow f$ and calls an $\mathrm{FL}_{e}$-algebra involutive if $\left(x^{\prime}\right)^{\prime}=x$ holds. An involutive $\mathrm{FL}_{e}$-algebra is called odd if the residual complement operation 
leaves the unit element fixed, that is, $t^{\prime}=t$, and even if the following (two) quasi-identities hold: $x<t \Leftrightarrow x \leq f$. The former condition is equivalent to $f=t$, while the latter quasi-identities are equivalent to assuming that $f$ is the lower cover of $t$ (and $t$ is the upper lower cover of $f$ ) if chains (or more generally conic algebras) are considered. An $\mathrm{FL}_{e}$-algebra is called integral if the unit element of the multiplication is the top element of its universe. Commutative residuated lattices are the $f$-free reducts of $\mathrm{FL}_{e}$-algebras. The geometric meaning of $t^{\prime}=f$, which is valid in all involutive $\mathrm{FL}_{e}$-algebras, is that the two constants are positioned symmetrically inside the underlying set. Therefore, one extremal setting is the integral case, when $t$ and $f$ are the top and bottom elements of $X$, respectively, and the other extremal one is the even or odd case when the two constants are both "in the middle of $X$ ". A bottom-top setup is not possible: if a residuated lattice have a bottom element then that element is annihilating, hence cannot be the unit of the multiplication ${ }^{3}$. Both odd or even involutive $\mathrm{FL}_{e}$-chains and IMTLchains are involutive residuated chains with a single additional postulate: the integrality condition of IMTL-chains postulates the unit element to be in one of its possible extremal positions (top), whereas the odd or even condition postulates the unit element to be in its other extremal position (in the middle).

Section 3 contains results on the local unit element function of involutive $\mathrm{FL}_{e}$-algebras, a key concept for our representation theorem. Even involutive $\mathrm{FL}_{e}$-algebras with non-idempotent and idempotent falsum constants will be characterized with respect to odd involutive $\mathrm{FL}_{e}$-algebras in Sections 5 and 6 , respectively. In Section 4 even and odd involutive $\mathrm{FL}_{e}$-chains will be partitioned into their so-called layer algebras, which are also even or odd involutive $\mathrm{FL}_{e}$-chains but more specific ones than the original algebra in the sense that they are either cancellative or are close to being such: in the latter case there exists a canonical homomorphism which maps the layer algebra into a cancellative one from which the layer algebra can be uniquely recovered. This specificity allows for establishing a connection between them and abelian o-groups in Section 7, by using the characterizations of Sections 5 and 6. These lead to the main result of the paper in Section 8: a one-to-one correspondence in a constructive manner between the class of all even or odd involutive commutative residuated chains and the class of bunches of layer groups. Layer groups are direct systems of abelian o-groups equipped with further structure.

\footnotetext{
${ }^{3}$ Unless the algebra is trivial.
} 


\section{The Local Unit Element Function}

Let $(X, \leq)$ be a poset. For $x, y \in X$ we say that $y$ is a cover of $x$ if $y>x$ and there exists no $z \in X$ such that $y>z>x$. For $x \in X$ let $x_{\uparrow}$ to be the unique cover of $x$ if such exists, and let $x_{\uparrow}=x$ otherwise. Define $x_{\downarrow}$ dually. Call $\downarrow$ and $\uparrow$ the neighbour operations of $(X, \leq)$. A partially ordered algebra with a poset reduct is called discretely ordered if for any element $x$, $x_{\downarrow}<x<x_{\uparrow}$ holds. If ${ }^{\prime}$ is an order-reversing involution of $X$ then it holds true that

$$
x_{\uparrow}^{\prime}=\left(x_{\downarrow}\right)^{\prime} \quad \text { and } \quad x_{\downarrow}^{\prime}=\left(x_{\uparrow}\right)^{\prime} .
$$

Algebras will be denoted by bold capital letters, their underlying sets by the same regular letter unless otherwise stated. Sometimes the lattice operators of an $\mathrm{FL}_{e}$-algebra will be replaced by their induced ordering $\leq$ in the signature, in particular, if an $\mathrm{FL}_{e^{-}}$chain is considered, that is, if the ordering is total. Call the elements $x \geq t$ of an $\mathrm{FL}_{e}$-algebra $\mathbf{X}=(X, \wedge, \vee, \cdot, \rightarrow, t, f)$ positive. Call $\mathbf{X}$ conic if all elements of $X$ are comparable with $t$. Assume $\mathbf{X}$ is involutive. For $x \in X$ let

$$
\tau(x)=x \rightarrow x,
$$

or equivalently, define $\tau(x)$ to be the greatest element of $\operatorname{Stab}_{x}=\{z \in$ $X \mid z x=x\}$. A key step toward our representation theorem is to understand the role of the $\tau$ function. In investigating more specific odd involutive $\mathrm{FL}_{e^{-}}$ algebras $\tau(x)$ was recognized to play the role of the 'absolute value of $x$ ' and was denoted by $|x|$, see [50]. The definition of layers in (3.2) and Lemma 3.1 reveal the true nature of $\tau$ in the present, more general setting: $\tau$ can be view as a 'local unit element' function. For any positive idempotent element $u$ define

$$
X_{u}=\{x \in X: \tau(x)=u\}
$$

and call it the $u$-layer of $\mathbf{X}$. The $X_{u}$ 's form a partition of $X$ by claim (vii) below, and if $x \in X_{u}$ then $\tau(x)$ is the unit element for the subset $X_{u}$ of $X$ by claim $(\mathrm{v})^{4}$. Corresponding to the rotation-annihilation construction the idea of decomposing a class of $\mathrm{FL}_{e}$-algebras with the help of a closely related

\footnotetext{
${ }^{4}$ For a first glance it might occur to the reader that the definition of $X_{u}$ is equivalent to the well-known way of localizing in semigroup theory, namely, for an idempotent $u$, to the universe $M_{u}=\{x \in X: x u=x\}$ of the greatest unitary subsemigroup of $\mathbf{X}$ with unit $u$. It is not the case if $u>t$. Rather, $X_{u}$ can be regarded as the "outer edge" of $M_{u}$ in the following sense: it holds true that $M_{u}=\bigcup_{v \leq u} X_{v}$ and $X_{u}=M_{u} \backslash \bigcup_{v<u} X_{v}(u$ and $v$ are positive idempotents).
} 
notion, the skeleton function of the algebra ${ }^{5}$ has been presented already in [27]. The name local unit function and the corresponding idea of using it to localize elements of the algebra has been introduced in [31], and the way of localizing as done in (3.2) is being introduced in the present paper, see also [29]. Some of the statements of the following lemma can be found in [31] or in [19], too. We include their proofs to keep the paper self-contained. As usual, when multiplication is denoted by $\cdot$, we write $x y$ instead of $x \cdot y$.

LEMma 3.1. Let $\mathbf{X}=(X, \wedge, \vee, \cdot, \rightarrow, t, f)$ be an involutive $F L_{e}$-algebra. The following statements hold true.

(i) $t^{\prime}=f$,

(ii) $x \rightarrow y=\left(x y^{\prime}\right)^{\prime}$,

(iii) if $t \geq f$ then $x y \leq\left(x^{\prime} y^{\prime}\right)^{\prime}$,

(iv) if the algebra is conic and either even or $t \leq f$ then $y_{1}>y$ implies $\left(x^{\prime} y^{\prime}\right)^{\prime} \leq x y_{1}$

(v) $\tau(x) x=x$ and $\tau(x) \geq t$,

(vi) $u \geq t$ is idempotent if and only if $\tau(u)=u$,

(vii) $\{\tau(x): x \in X\}$ is equal to the set of positive idempotent elements of $\mathbf{X}$

(viii) $x_{1} y>x y$ holds whenever $x_{1}>x$ and $y$ is invertible,

(xi) $\tau(x)=\tau\left(x^{\prime}\right)$,

(x) if $\cdot$ is cancellative then for $x \in X, x x^{\prime}=f$,

(xi) if $\mathbf{X}$ is odd then the $(X, \wedge, \vee, \cdot, t)$-reduct of $\mathbf{X}$ is a lattice-ordered abelian group if and only if $\cdot$ is cancellative, ${ }^{6}$

(xii) for $x \geq t, \tau(x) \leq x$ holds,

(xiii) if $\mathbf{X}$ is an odd chain then $X_{t}$ contains all the invertible elements of $X$, and it is a universe of an abelian o-group, its inverse operation is the residual complement operation.

(xvi) if $\mathbf{X}$ is an odd or even, cancellative, discretely ordered chain then $z t_{\downarrow}=$ $z_{\downarrow}$

\footnotetext{
${ }^{5}$ The skeleton function was defined there as the residual complement of what we coined local-unit function.

${ }^{6}$ When we (loosely) speak about a subgroup of odd involutive $\mathrm{FL}_{e}$-algebra $\mathbf{X}$ in the sequel, we shall mean a cancellative subalgebra of $\mathbf{X}$.
} 
$(\mathrm{xv})$ if $\mathbf{X}$ is an odd or even chain then $x y<x_{1} y_{1}$ holds whenever $x<x_{1}$ and $y<y_{1}$,

(xvi) if $\mathbf{X}$ is an odd or even chain and $A$ is an $\mathbf{X}$-term which contains only the operations $\cdot \rightarrow$ and ' then for any evaluation e of the variables of $A$ into $X, \tau(e(A))$ equals the maximum of the $\tau$-values of the variables and constants of $A$ under $e$,

\section{ProOF.}

(i) Obvious by residuation.

(ii) Using that ' is an involution one obtains $\left(x y^{\prime}\right)^{\prime}=\left(x y^{\prime}\right) \rightarrow f=x \rightarrow$ $\left(y^{\prime} \rightarrow f\right)=x \rightarrow y$ (folklore).

(iii) Next, $(x y)\left(x^{\prime} y^{\prime}\right)=[x(x \rightarrow f)][y(y \rightarrow f)] \leq f f \leq t f=f$, hence $x y \leq$ $\left(x^{\prime} y^{\prime}\right) \rightarrow f$ follows by adjointness.

(iv) Since the algebra is conic and involutive, every element is comparable with $f$, too. Indeed, if for any $a \in X, a$ were not comparable with $f$ then, since ' is an order reversing involution, $a^{\prime}$ were not comparable with $f^{\prime}$, and $f^{\prime}=t$ since the algebra is involutive, a contradiction. Therefore, by residuation, $y_{1}>\left(y^{\prime}\right)^{\prime}=y^{\prime} \rightarrow f$ implies $y_{1} y^{\prime} \not \leq f$, that is, $y_{1} y^{\prime}>f$. If $t \leq f$ then $y_{1} y^{\prime} \geq t$ follows. Likewise, if the algebra is even then $y_{1} y^{\prime} \not \leq f$ implies $y_{1} y^{\prime} \nless t$, that is, $y_{1} y^{\prime} \geq t$, since the algebra is conic. Therefore, $\left(x y_{1}\right)^{\prime}=\left(x y_{1}\right)^{\prime} t \leq\left(x y_{1}\right)^{\prime} y_{1} y^{\prime} \stackrel{(i i)}{=}\left(y_{1}\left(y_{1} \rightarrow x^{\prime}\right)\right) y^{\prime} \leq$ $x^{\prime} y^{\prime}$ follows.

(v) Since $t$ is the unit element, $S t a b_{x}$ is nonempty. Therefore, by residuation $\tau(x)=x \rightarrow x$ is its largest element, hence $\tau(x) x=x$ holds. Since $t x=x, \tau(x) \geq t$ follows by residuation.

(vi) If $u \geq t$ is idempotent then from $u u=u, u \rightarrow u \geq u$ follows by adjointness. But for any $z>u, u z \geq t z=z>u$, hence $\tau(u)=u$ follows. On the other hand, by claim $(\mathrm{v}), \tau(u)=u$ implies $u \geq t$, and also the idempotency of $u$ follows since $u u=u \tau(u)=u$.

(vii) If $u>t$ is idempotent then claim (vi) shows that $u$ is in the range of $\tau$. If $u$ is in the range of $\tau$, that is $\tau(x)=u$ for some $x \in X$ then if $\tau(\tau(x))=\tau(x)$ then it implies $\tau(u)=u$, hence $u$ is a positive idempotent element by claim (vi), and we are done. Hence it suffices to prove $\tau(\tau(x))=\tau(x)$ for all $x$. By claim (ii), $x \rightarrow x=\tau(x)$ is equivalent to $x x^{\prime}=\tau(x)^{\prime}$. Hence, $\tau(x) \tau(x)^{\prime}=\tau(x)\left(x x^{\prime}\right)=(\tau(x) x) x^{\prime} \stackrel{(v)}{=} x x^{\prime}=$ $\tau(x)^{\prime}$ follows, which is equivalent to $\tau(\tau(x))=\tau(x) \rightarrow \tau(x)=\tau(x)$. 
(viii) $x_{1} y \geq x y$ holds by monotonicity, and if $x_{1} y=x y$ then $x_{1}=x_{1} y y^{-1}=$ $x y y^{-1}=x$, a contradiction.

(ix) By claim (ii) and the involutivity of ',$\tau(x)=x \rightarrow x=\left(x x^{\prime}\right)^{\prime}=$ $\left(x^{\prime} x^{\prime \prime}\right)^{\prime}=x^{\prime} \rightarrow x^{\prime}=\tau\left(x^{\prime}\right)$.

(x) Since $\cdot$ is cancellative, the strictly increasing nature of · clearly follows: if $u<v$ then $u w<v w$. Therefore, $x a>x t=x$ for any $a>t$, and hence $x \rightarrow x=t$. An application of claim (ii) ends the proof.

(xi) Necessity is straightforward, sufficiency follows from claim (x) since $f=t$.

(xii) For $x \geq t, x \stackrel{(v)}{=} x \tau(x) \geq t \tau(x)=\tau(x)$.

(xiii) If $x \in X_{t}$ then $\tau(x)=x \rightarrow x=t$ and hence $x x^{\prime} \stackrel{(i i)}{=} t^{\prime} \stackrel{(i)}{=} f=t$ holds since the algebra is odd. This shows that all elements of $X_{t}$ are invertible in $X$ and the inverse operation is '. On the other hand, if $x \in X$ is invertible, that is, $x y=t$ for some $y \in X$ then $\tau(x)=t$ : for every $z>t$ it holds true that $x z>x$ since the opposite, that is, $x z=x$ would imply $z=t z=(y x) z=y(x z)=y x=t$, a contradiction. Invertible element are clearly closed under · and '. Since the order is total, the meet and the join of two invertible elements are also invertible.

(xiv) Since · is cancellative, · is strictly increasing; we shall use it without further mention. Now, $z t_{\downarrow}<z t=z$ holds since $t_{\downarrow}<t$ follows from the algebra being discretely ordered. Contrary to the statement, assume that there exists $a$ such that $z t_{\downarrow}<a<z$. Multiplying with $z^{\prime}, f t_{\downarrow}<$ $a z^{\prime}<f$ follows by claim (x). In the odd case it yields $t_{\downarrow}<a z^{\prime}<t$, a contradiction to the definition of $\downarrow$. In the even case it yields $t_{\downarrow} t_{\downarrow}<$ $a z^{\prime}<t_{\downarrow}$. Since $t_{\downarrow}=t t_{\downarrow}<t_{\uparrow} t_{\downarrow}<t_{\uparrow} t=t_{\uparrow}$, we obtain $t_{\uparrow} t_{\downarrow}=t$, and hence multiplication by $t_{\uparrow}$ implies $t_{\downarrow}<t_{\uparrow} a z^{\prime}<t$, a contradiction to the definition of $\downarrow$.

(xv) By claim (iii), $\left(x^{\prime} y^{\prime}\right)^{\prime} \geq x y$ holds, hence it suffices to prove $x_{1} y_{1}>$ $\left(x^{\prime} y^{\prime}\right)^{\prime}$. Assume the opposite, which is $x_{1} y_{1} \leq\left(x^{\prime} y^{\prime}\right)^{\prime}$ since $(X, \leq)$ is a chain. By adjointness we obtain $\left(x^{\prime} x_{1}\right)\left(y^{\prime} y_{1}\right)=\left(x_{1} y_{1}\right)\left(x^{\prime} y^{\prime}\right) \leq f$, and from $x_{1}>x=\left(x^{\prime}\right)^{\prime}, x^{\prime} x_{1}>f$ follows by residuation since $(X, \leq)$ is a chain.

In the odd case these reduce to $\left(x^{\prime} x_{1}\right)\left(y^{\prime} y_{1}\right) \leq t$ and $x^{\prime} x_{1}>t$. Analogously we obtain $y^{\prime} y_{1}>t$. Therefore $\left(x^{\prime} x_{1}\right)\left(y^{\prime} y_{1}\right) \geq\left(x^{\prime} x_{1}\right) t=x^{\prime} x_{1}>t$ 
follows, a contradiction.

In the even case these reduce to $\left(x^{\prime} x_{1}\right)\left(y^{\prime} y_{1}\right)<t$ and $x^{\prime} x_{1} \geq t$. Analogously we obtain $y^{\prime} y_{1} \geq t$. Therefore $\left(x^{\prime} x_{1}\right)\left(y^{\prime} y_{1}\right) \geq t t=t$ follows, a contradiction.

(xvi) We have already seen $\tau(x)=\tau\left(x^{\prime}\right)$ in claim (ix).

Next, we claim $\tau(x y)=\max (\tau(x), \tau(y))\left(=\tau(x) \tau(y)^{7}\right)$. Indeed, $\tau(x y) \geq$ $\tau(x)$ holds by residuation since $x y \rightarrow x y \geq x \rightarrow x$ is equivalent to $y x(x \rightarrow x) \leq x y$. Assume $z:=\tau(x y)>\max (\tau(x), \tau(y))$. Since $\tau$ assigns to $x$ the greatest element of the stabilizer set of $x$, therefore $z>\max (\tau(x), \tau(y))$ implies that $z$ does not stabilize $x$ neither $y$, hence $x<z x$ and $y<z y$ holds by the monotonicity of $\cdot$. On the other hand, $(z x)(z y)=((x y) z) z \stackrel{(v)}{=} x y$ follows, a contradiction to claim (xv). This settles the claim.

By claim (ii), any term which contains only the connectives $\cdot, \rightarrow$ and ' can be represented by an equivalent term using the same variables and constants but containing only · and '. An easy induction on the recursive structure of this equivalent term using the two claims above concludes the proof.

The next lemma states that for an odd involutive $\mathrm{FL}_{e}$-algebra, it is exactly cancellativity which is needed to make it a lattice ordered group.

LEMMA 3.2.

(1) For a cancellative odd involutive $F L_{e}$-algebra

$$
\mathbf{X}=(X, \wedge, \vee, \cdot, \rightarrow, t, t)
$$

with residual complement ${ }^{\prime}, \lambda(\mathbf{X})=\left(X, \wedge, \vee, \cdot,{ }^{-1}, t\right)$ is a lattice-ordered abelian group, called the lattice-ordered abelian group induced by $\mathbf{X}$, where

$$
x^{-1}=x^{\prime}=x \rightarrow t,
$$

(2) For a lattice-ordered abelian group

$$
\boldsymbol{G}=\left(G, \leq, \cdot,^{-1}, t\right),
$$

\footnotetext{
${ }^{7}$ For positive idempotents $u \leq v$ it holds true that $u v=\max (u, v)$ since $v=t v \leq u v \leq$ $v v=v$.
} 
$\iota(\boldsymbol{G})=(G, \wedge, \vee, \cdot, \rightarrow, t, t)$ is a cancellative odd involutive $F L_{e}$-algebra, called the cancellative odd involutive $F L_{e}$-algebra induced by $\boldsymbol{G}$, where

$$
\begin{aligned}
x \rightarrow y & =x^{-1} y, \\
x^{\prime} & =x^{-1},
\end{aligned}
$$

(3) With the above assumptions it holds true that $\iota(\lambda(\mathbf{X}))=\mathbf{X}$ and $\lambda(\iota(\boldsymbol{G}))$ $=G$.

Proof. Claim (xi) in Lemma 3.1 confirms the first statement, the rest is folklore or obvious.

The following lemma will simplify the proof of Theorem 6.3 and Lemma 4.2 . Let $\mathcal{M}=(M, \leq, \cdot)$ be a structure such that $(M, \leq)$ is a poset and $(M, \cdot)$ is a commutative semigroup. Call $c \in M$ a dualizing element ${ }^{8}$ of $\mathcal{M}$, if (i) for $x \in M$ there exists, $x \rightarrow c^{9}$ and (ii) for $x \in M,(x \rightarrow c) \rightarrow c=x$.

LEMMA 3.3. If there exists a dualizing element $c$ of $\mathcal{M}$ then $\cdot$ is residuated and its residual operation is given by $x \rightarrow y=(x(y \rightarrow c)) \rightarrow c$.

Proof. $z x \leq y$ is equivalent to $z x \leq(y \rightarrow c) \rightarrow c$. By adjointness it is equivalent to $(z x)(y \rightarrow c) \leq c$. By associativity it is equivalent to $z(x(y \rightarrow c)) \leq c$, which is equivalent to $z \leq(x(y \rightarrow c)) \rightarrow c$ by adjointness. By residuation $x \rightarrow y=(x(y \rightarrow c)) \rightarrow c$ follows.

\section{Odd and Even Involutive $\mathrm{FL}_{e}$-chains vs. Bunches of Layer Algebras}

We shall prove the main theorem of the paper for three different kinds of involutive $\mathrm{FL}_{e}$-chains: for odd involutive $\mathrm{FL}_{e}$-chains, for even involutive $\mathrm{FL}_{e}$-chains with an idempotent falsum, and for even involutive $\mathrm{FL}_{e}$-chains with a non-idempotent falsum.

Definition 4.1. Let $\left(\kappa, \leq_{\kappa}\right)$ be a totally ordered set with least element $t$, and let an ordered triple $\left\langle\bar{\kappa}_{I}, \bar{\kappa}_{J},\{t\}\right\rangle$ be a partition of $\kappa$, where $\bar{\kappa}_{I}$ and $\bar{\kappa}_{J}$ can also be empty. Define $\kappa_{o}, \kappa_{J}$, and $\kappa_{I}$ by one of the rows of Table $1 .{ }^{10}$

\footnotetext{
${ }^{8}$ Dualizing elements have been defined only in residuated structures in the literature, see e.g. [19, Section 3.4.17.].

${ }^{9}$ That is, the exists the greatest element of the set $\{z \in M \mid x z \leq c\}$.

${ }^{10}$ Explanation: if the first, the second, or the third row is used then the algebra corresponding to the bunch will be odd, even with a non-idempotent falsum constant, or even with an idempotent falsum constant, respectively.
} 
Table 1. Three cases - three classes

\begin{tabular}{lll}
\hline$\kappa_{o}$ & $\kappa_{J}$ & $\kappa_{I}$ \\
\hline$\{\mathrm{t}\}$ & $\bar{\kappa}_{J}$ & $\bar{\kappa}_{I}$ \\
$\emptyset$ & $\bar{\kappa}_{J} \cup\{t\}$ & $\bar{\kappa}_{I}$ \\
$\emptyset$ & $\bar{\kappa}_{J}$ & $\bar{\kappa}_{I} \cup\{t\}$ \\
\hline
\end{tabular}

Let $\mathbf{X}_{u}=\left(X_{u}, \leq_{u},,{ }^{\cdot} u, \rightarrow_{u}, u, u^{\prime}\right)$ be a family of involutive $\mathrm{FL}_{e}$-chains indexed by elements of $\kappa$ (let ${ }^{\mu}$ denote the residual complement operation, $\downarrow u$ and $\uparrow_{u}$ the neighbour operations of $\mathbf{X}_{u}$ ), such that $\mathbf{X}_{u}$ is

$\begin{cases}\text { cancellative and odd } & \text { if } u \in \kappa_{O} \\ \text { discretely ordered, cancellative and even } & \text { if } u \in \kappa_{J}(4.1) \\ \text { even with an idempotent falsum satisfying } x \cdot{ }_{u} x^{{ }^{u}}=u^{u^{\prime}} & \text { if } u \in \kappa_{I}\end{cases}$

11 and such that for $u, v \in \kappa, u \leq_{\kappa} v$, there exist a

$$
\text { homomorphism } \rho_{u \rightarrow v}
$$

from the residuated lattice reduct of $\mathbf{X}_{u}$ to the residuated lattice reduct of $\mathbf{X}_{v}$ satisfying

(A1) $\rho_{u \rightarrow u}=i d_{X_{u}}$ and $\rho_{v \rightarrow w} \circ \rho_{u \rightarrow v}=\rho_{u \rightarrow w} \quad$ (direct system property), (A2) $\rho_{u \rightarrow v}(u)=\rho_{u \rightarrow v}\left(u^{\prime}\right)^{12} \quad$ (constants' collision condition).

Call $\mathcal{A}=\left\langle\mathbf{X}_{u}, \rho_{u \rightarrow v}\right\rangle_{\left\langle\kappa_{o}, \kappa_{J}, \kappa_{I}, \leq_{\kappa}\right\rangle}$ a bunch of layer algebras. Call the $\mathbf{X}_{u}$ 's the layer algebras, call $\left\langle\kappa, \leq_{\kappa}\right\rangle$ the skeleton, call $\left\langle\kappa_{o}, \kappa_{J}, \kappa_{I}\right\rangle$ the partition of the skeleton, and call $\left\langle\mathbf{X}_{u}, \rho_{u \rightarrow v}\right\rangle_{\kappa}$ the direct system of layer algebras over $\kappa$. Note that $\kappa$ can be recovered from its partition, (and ultimately, from $\mathcal{A}$ ) via $\kappa=\kappa_{o} \cup \kappa_{J} \cup \kappa_{I}$.

We prove that every odd or even involutive $\mathrm{FL}_{e}$-chain can be represented by a unique bunch of layer algebras. Later, in Section 7 we prove that every bunch of layer algebras can be represented by a unique bunch of layer groups.

LEMmA 4.2. The following statements hold true.

(1) Given an odd or an even involutive $F L_{e}$-chain $\mathbf{X}=(X, \leq, \cdot, \rightarrow, t, f)$ with residual complement operation',

$$
\mathcal{A}_{\mathbf{X}}=\left\langle\mathbf{X}_{u}, \rho_{u \rightarrow v}\right\rangle_{\kappa}
$$

\footnotetext{
${ }^{11}$ Hence with a non-idempotent falsum.

${ }^{12}$ If $u \in \kappa_{o}$ then $\rho_{u \rightarrow v}(u)=\rho_{u \rightarrow v}\left(u^{\prime}\right)$ trivially holds since $t^{\dagger}=t$.
} 
is a bunch of layer algebras, called the bunch of layer algebras of $\mathbf{X}$, where $\tau(x)=x \rightarrow x, \kappa=\{\tau(x): x \in X\}, \leq_{\kappa}=\leq \cap(\kappa \times \kappa), \bar{\kappa}_{I}=\{u \in$ $\kappa \backslash\{t\}: u^{\prime}$ is idempotent $\}, \bar{\kappa}_{J}=\left\{u \in \kappa \backslash\{t\}: u^{\prime}\right.$ is not idempotent $\}$, $\kappa_{o}, \kappa_{J}, \kappa_{I}$ are defined by Table 2 ,

$\boldsymbol{\kappa}=\left\langle\kappa_{o}, \kappa_{J}, \kappa_{I}, \leq_{\kappa}\right\rangle$, for $u \in \kappa$,

$$
\mathbf{X}_{u}=\left(X_{u}, \leq_{u}, \cdot u, \rightarrow_{u}, u, u^{\prime}\right),
$$

where

$$
X_{u}=\{x \in X: \tau(x)=u\},
$$

$\leq_{u}, \cdot{ }_{u}$, and $\rightarrow_{u}$ are restrictions of $\leq, \cdot$, and $\rightarrow$ to $X_{u}$, for $x \in X_{u}$, $x^{\mu^{u}}=x \rightarrow u^{\prime}$, and for $u, v \in \kappa, u \leq_{\kappa} v, \rho_{u \rightarrow v}: X_{u} \rightarrow X_{v}$ is given by

$$
\rho_{u \rightarrow v}(x)=v x .
$$

(2) Given a bunch of layer algebras $\mathcal{A}=\left\langle\mathbf{X}_{u}, \rho_{u \rightarrow v}\right\rangle_{\boldsymbol{\kappa}}$ with $\boldsymbol{\kappa}=\left\langle\kappa_{o}, \kappa_{J}, \kappa_{I}\right.$, $\left.\leq_{\kappa}\right\rangle, \mathbf{X}_{u}=\left(X_{u}, \leq_{u}, \cdot_{u}, \rightarrow_{u}, u, u^{\mu}\right)$, and $x^{\mu^{\mu}}=x \rightarrow_{u} u^{\mu^{\mu}}$,

$$
\mathcal{X}_{\mathcal{A}}=\left(X, \leq, \cdot, \rightarrow, t, t^{\prime}\right)
$$

is an involutive $F L_{e}$-chain, called the involutive $F L_{e}$-chain derived from $\mathcal{A}$, where

$$
X=\bigcup_{u \in \kappa} X_{u}
$$

for $v \in \kappa, \rho_{v}: X \rightarrow X$ is defined by

$$
\rho_{v}(x)=\left\{\begin{array}{ll}
\rho_{u \rightarrow v}(x) & \text { if } u<_{\kappa} v \text { and } x \in X_{u} \\
x & \text { if } u \geq_{\kappa} v \text { and } x \in X_{u}
\end{array},\right.
$$

for short, for $x \in X_{u}$ and $y \in X_{v},{ }^{13}$

$$
\begin{aligned}
& x<y \text { iff } \rho_{u v}(x)<_{u v} \rho_{u v}(y) \text { or } \rho_{u v}(x)=\rho_{u v}(y) \text { and } u<_{\kappa} v \\
& x y=\rho_{u v}(x) \cdot{ }_{u v} \rho_{u v}(y) \\
& x^{\prime}=x^{{ }^{\prime}} \\
& x \rightarrow y=\left(x y^{\prime}\right)^{\prime}
\end{aligned}
$$

\footnotetext{
${ }^{13}$ Note that for $u, v \in \kappa, u v=\max _{\kappa}(u, v)$ since $u, v$ are positive idempotents.
} 
Table 2. Three cases - three classes

\begin{tabular}{llll}
\hline$\kappa_{o}$ & $\kappa_{J}$ & $\kappa_{I}$ & \\
\hline$\{\mathrm{t}\}$ & $\bar{\kappa}_{J}$ & $\bar{\kappa}_{I}$ & if $\mathbf{X}$ is odd \\
$\emptyset$ & $\bar{\kappa}_{J} \cup\{t\}$ & $\bar{\kappa}_{I}$ & if $\mathbf{X}$ is even and $f$ is not idempotent \\
$\emptyset$ & $\bar{\kappa}_{J}$ & $\bar{\kappa}_{I} \cup\{t\}$ & if $\mathbf{X}$ is even and $f$ is idempotent \\
\hline
\end{tabular}

14 and $t$ is the least element of $\kappa . \mathcal{X}_{\mathcal{A}}$ is odd if $t \in \kappa_{o}$, even with a non-idempotent falsum if $t \in \kappa_{J}$, and even with an idempotent falsum if $t \in \kappa_{I}$.

(3) For a bunch of layer algebras $\mathcal{A}, \mathcal{A}_{\left(\mathcal{X}_{\mathcal{A}}\right)}=\mathcal{A}$, and for an odd or even involutive $F L_{e}$-chain $\mathbf{X}, \mathcal{X}_{\mathcal{A}_{\mathbf{X}}}=\mathbf{X}$.

Proof. (1): $\kappa$ is the set of positive idempotent elements of $\mathbf{X}$ by claim (vii) in Lemma 3.1. Therefore, the least element of $\kappa$ is $t$, and $\kappa$, being a subset of $X$, is totally ordered. The ordered triple $\left\langle\bar{\kappa}_{I}, \bar{\kappa}_{J},\{t\}\right\rangle$ is clearly a partition of $\kappa$, where $\bar{\kappa}_{I}$ and $\bar{\kappa}_{J}$ can also be empty.

Let $u \in \kappa . X_{u}$ is nonempty since $u \in X_{u}$ holds by claim (vi) in Lemma 3.1, and, being a subset of $X, X_{u}$ is totally ordered by $\leq_{u}$. $X_{u}$ is closed under $\cdot{ }_{u}, \rightarrow_{u}$, and ' by claim (xvi) in Lemma 3.1, and thus $u^{{ }^{\prime}}=u \rightarrow u^{\prime} \in X_{u}$. Since $\tau(x)=u$ holds for $x \in X_{u}$, therefore $x u=x \tau(x) \stackrel{\text { L3.1. }}{=}(v)$ shows that $u$ is the unit element of $\mathbf{X}_{u}$. For $x \in X_{u}$,

$$
x^{\prime \prime}=x^{\prime}
$$

holds since $x^{\mu}=x \rightarrow u^{\prime}=x \rightarrow(u \rightarrow f) \stackrel{\text { is residuated }}{=}(x u) \rightarrow f=x \rightarrow f=x^{\prime}$. Therefore, $u^{{ }^{u}}=u^{\prime}$, and hence $x^{{ }^{u}}=x \rightarrow u^{{ }^{u}}$. Summing up,

$$
\mathbf{X}_{u}=\left(X_{u}, \leq_{u}, \cdot_{u}, \rightarrow_{u}, u, u^{\prime}\right) \text { is an involutive } \mathrm{FL}_{e} \text {-chain. }
$$

Next we prove that the $\mathbf{X}_{u}$ 's satisfy (4.1).

If $u \in \kappa_{o}$ then $\mathbf{X}$ is odd by Table 2. By claim (xiii) in Lemma 3.1 and by Lemma 3.2, $\mathbf{X}_{u}$ is a cancellative odd involutive $\mathrm{FL}_{e}$-chain.

If $u \in \kappa_{J}$ then by Table $2, u=t$ or $u \in \bar{\kappa}_{J}$. In both cases $u^{\prime}$ is not idempotent.

We prove that $X_{u}$ is discretely ordered by showing

$$
x u^{\prime}=x_{\downarrow u}<x
$$

\footnotetext{
${ }^{14}$ Alternatively, we may write $x \leq y$ iff $\rho_{u v}(x) \leq_{u v} \rho_{u v}(y)$ except if $u>_{\kappa} v$ and $\rho_{u v}(x)=\rho_{u v}(y)$.
} 
for $x \in X_{u}$, where $\downarrow_{u}$ denotes the neighbour operation on $X_{u}$. It holds true that

$$
u^{\prime}<t
$$

Indeed, if $u \in \bar{\kappa}_{J}$ then $u>t$ and the involutivity of ' on $X$ implies $u^{\prime}<$ $t^{\prime} \stackrel{L 3.1(i)}{=} f \stackrel{\mathrm{X}}{\text { is odd or even }} \mathrm{s}$, whereas if $u=t$ then $u^{\prime}=t^{\prime}<t$ since the second row of Table 2 shows that $\mathbf{X}$ is even. Therefore, by denoting $y=u^{\prime} u^{\prime}$,

$$
y<u^{\prime}
$$

holds since $y=u^{\prime} u^{\prime} \stackrel{(4.14)}{\leq} u^{\prime} t=u^{\prime}$ and equality cannot not hold since $u^{\prime}$ is not idempotent. Now $x u^{\prime} \stackrel{(4.14)}{\leq} x t=x$ follows. Assume, by contradiction $x u^{\prime}=x$. It would imply $x y=x\left(u^{\prime} u^{\prime}\right)=\left(x u^{\prime}\right) u^{\prime}=x$, hence by claim (iii) in Lemma 3.1, $\left(x^{\prime} y^{\prime}\right)^{\prime} \geq x y=x$, and in turn $x^{\prime} y^{\prime} \leq x^{\prime}$ would follow on the one hand. On the other hand, from $y^{\prime} \stackrel{(4.15)}{>} u \geq t$, by monotonicity $x^{\prime} y^{\prime} \geq x^{\prime} t=x^{\prime}$ follows, thus we obtain $x^{\prime} y^{\prime}=x^{\prime}$, and hence $y^{\prime} \leq \tau\left(x^{\prime}\right) \stackrel{L 3.1(i x)}{=} u$, a contradiction to (4.15). We have just seen that $x u^{\prime}<x$. Next, assume that there exists $z \in X_{u}$ such that $x u^{\prime}<z<x$ holds. Since $z<x, x u^{\prime} \geq\left(z^{\prime} u\right)^{\prime}=z^{\prime \prime}=z$ follows by claim (iv) in Lemma 3.1, a contradiction, so (4.13) is confirmed.

Next we show that $\mathbf{X}_{u}$ is even: $u^{\prime} \stackrel{(4.11)}{=} u^{\prime}=u u^{\prime} \stackrel{(4.13)}{=} u_{\downarrow_{u}}<u$.

Finally, we show that $\mathbf{X}_{u}$ is cancellative, by showing that every element of $X_{u}$ has inverse, that is, for $x \in X_{u}, x \cdot{ }_{u} x_{\uparrow_{u}}^{\mu^{\mu}}=u$. On the one hand, referring to (4.12), $x \cdot{ }_{u} x_{\uparrow_{u}}^{\mu}>_{u} u^{\mu}$ holds by residuation since $X$ is a chain. It is equivalent to $x \cdot{ }_{u} x_{\uparrow_{u}}^{u^{u}} \geq_{u} u$ since $\mathbf{X}_{u}$ is even, yielding $x \cdot{ }_{u} x_{\uparrow_{u}}^{u} \geq u$. On the other hand, $x \cdot \cdot_{u} x_{\uparrow_{u}}^{u_{u}}=x x_{\uparrow_{u}}^{u^{u}} \stackrel{(4.11)}{=} x x^{\prime} \uparrow_{u} \stackrel{(3.1)}{=} x\left(x_{\downarrow_{u}}\right)^{\prime} \stackrel{(4.13)}{=} x\left(x u^{\prime}\right)^{\prime} \stackrel{\text { L3.1(ii) }}{=}$ $x(x \rightarrow u) \stackrel{\text { is residuated }}{\leq} u$.

Summing up, $\mathbf{X}_{u}$ is a discretely ordered cancellative even involutive $\mathrm{FL}_{e^{-}}$ chain.

If $u \in \kappa_{I}$ then by Table $2, u=t$ or $u \in \bar{\kappa}_{I}$. In both cases $u^{\prime}$ is idempotent. We show that $\mathbf{X}_{u}$ is even: first, $u^{u}<u$ holds since if $u \in \bar{\kappa}_{I}$ then $u>t$ and hence $u^{\prime} \stackrel{(4.11)}{=} u^{\prime}<t^{\prime} \stackrel{\text { L3.1(i) }}{=} f \stackrel{\mathbf{X} \text { is odd or even }}{\leq} t<u$, whereas if $u=t$ then $\mathbf{X}$ is 
even by the third row of Table 2 and $u^{\prime} \stackrel{(4.11)}{=} u^{\prime}=t^{\prime} \stackrel{\text { L3.1(i) }}{=} f \stackrel{\mathrm{X} \text { is even }}{<} t=u$, and second, by claims (xii) and (xvi) in Lemma 3.1, no element $x \in X$ such that $u^{\prime}<x<u$ can be in $X_{u}$ (if $u^{\prime}<x<u$ then $u^{\prime}<x^{\prime}<u$, so we may safely assume $x \geq t$, and then $x \in X_{u}$ implies $u=\tau(x) \leq x<u$, a contradiction); thus $u^{\prime}=u_{\downarrow_{u}}$. Summing up, $\mathbf{X}_{u}$ is an even involutive $\mathrm{FL}_{e}$-chain with an idempotent falsum. It remains to prove $x \cdot{ }_{u} x^{{ }^{u}}=u^{{ }^{\prime}}$ for $x \in X_{u}$, which follows from $u^{\prime} \stackrel{(4.11)}{=} u^{\prime}=\tau(x)^{\prime}=(x \rightarrow x)^{\prime} \stackrel{\text { L3.1(ii) }}{=} x x^{\prime} \stackrel{(4.11)}{=}$ $x x^{{ }^{u}}=x \cdot{ }_{u} x^{{ }^{\prime}}$.

Next we prove that $\rho_{u \rightarrow v}$ is a homomorphism from the residuated lattice reduct of $\mathbf{X}_{u}$ to the residuated lattice reduct of $\mathbf{X}_{v}$. Let $u, v \in X$ be positive idempotent elements of $\mathbf{X}$ such that $u<v . \rho_{u \rightarrow v} \operatorname{maps} X_{u}$ to $X_{v}$ by claim (xvi) in Lemma 3.1. $\rho_{u \rightarrow v}$ preserves the ordering since $\cdot$ is monotone. $\rho_{u \rightarrow v}$ preserves products since $\cdot$ is associative and $v$ is idempotent.

To show that $\rho_{u \rightarrow v}$ preserves the residual operation we proceed as follows. Let $x, y \in X_{u}$. It holds true that $v\left(x y^{\prime}\right)^{\prime} x(v y)^{\prime} \stackrel{\text { L3.1(ii) }}{=} x(x \rightarrow y)\left(y \rightarrow v^{\prime}\right) v \leq f$ since $\cdot$ is residuated, hence by adjointness, $v\left(x y^{\prime}\right)^{\prime} \leq\left(x(v y)^{\prime}\right)^{\prime}$ follows. On the other hand, $v\left(x y^{\prime}\right)^{\prime} \stackrel{L 3.1(i i i)}{\geq} v\left(x^{\prime} y\right)=x^{\prime}(v y)$. Now $x=t x \leq v x$, and since $v>u=\tau(x)$ and hence $v$ does not stabilize $x, x<v x$ follows. Therefore, $x^{\prime}(v y) \stackrel{L 3.1(i v)}{\geq}\left((v x)(v y)^{\prime}\right)^{\prime}=\left(x\left(v(v y)^{\prime}\right)\right)^{\prime} \stackrel{(v y)^{\prime} \in X_{v}}{=}\left(x(v y)^{\prime}\right)^{\prime}$. Summing up, $v\left(x y^{\prime}\right)^{\prime}=\left(x(v y)^{\prime}\right)^{\prime}$. Therefore, $\rho_{u \rightarrow v}(x \rightarrow u y) \stackrel{\text { L3.1(ii) }}{=} v\left(x \cdot u y^{\mu^{\prime}}\right)^{\prime} \stackrel{(4.11)}{=}$ $v\left(x y^{\prime}\right)^{\prime}=\left(x(v y)^{\prime}\right)^{\prime} \stackrel{(v y)^{\prime} \in X_{v}}{=}\left(x\left(v(v y)^{\prime}\right)\right)^{\prime}=\left((v x)(v y)^{\prime}\right)^{\prime} \stackrel{(4.11)}{=}\left((v x) \cdot v(v y)^{\prime}\right)^{\prime \prime}$ $\stackrel{\text { L3.1(ii) }}{=}(v x) \rightarrow_{v}(v y)=\rho_{u \rightarrow v}(x) \rightarrow_{v} \rho_{u \rightarrow v}(y)$ an we are done.

Finally, $\rho_{u \rightarrow v}$ preserves the unit element:

$$
\rho_{u \rightarrow v}(u)=v u=v
$$

holds since $v=v v \geq v u \geq v t=v$. Summing up, $\rho_{u \rightarrow v}$ is a homomorphism from the residuated lattice reduct of $\mathbf{X}_{u}$ to the residuated lattice reduct of $\mathbf{X}_{v}$.

To conclude the proof of claim (1) it only remains to prove

(A1) For $u, v, w \in X$ positive idempotent elements such that $u \leq v \leq w$, and for $x \in X_{u}, \rho_{u \rightarrow u}(x)=u x \stackrel{\text { L3.1 }}{=}(v)$ and $\left(\rho_{v \rightarrow w} \circ \rho_{u \rightarrow v}\right)(x) \stackrel{(4.4)}{=}$ $w(v x)=(w v) x \stackrel{(4.16)}{=} w x=\rho_{u \rightarrow w}(x)$. 
(A2) Let $u, v \in X$ positive idempotent elements such that $u<v$. By claim (iv) in Lemma 3.1, v $=v t \geq v u^{\prime} \geq\left(v^{\prime} v\right)^{\prime}=\tau(v)=v$, yielding $\rho_{u \rightarrow v}\left(u^{\prime}\right) \stackrel{(4.4)}{=} v u^{\prime}=v \stackrel{(4.16)}{=} v u \stackrel{(4.4)}{=} \rho_{u \rightarrow v}(u)$.

(2): We conclude by a series of claims.

$\leq$ is a total ordering on $X$.

- Irreflexivity of $<$ is immediate form the irreflexivity of $<_{\kappa}$.

- Connectedness of $<$ is obvious, too: either $\rho_{u v}(x)<_{u v} \rho_{u v}(y)$ or $\rho_{u v}(y)$ $<_{u v} \rho_{u v}(x)$ or $\rho_{u v}(x)=\rho_{u v}(y)$ holds, since $\leq_{u v}$ is total. The first two (4.7) (4.7)

cases yield $x \stackrel{(4.7)}{<} y$ and $y \stackrel{(4.7)}{<} x$, respectively. If $\rho_{u v}(x)=\rho_{u v}(y)$ then either $u<_{\kappa} v$ or $v<_{\kappa} u$ or $u={ }_{\kappa} v$ holds since $\leq_{\kappa}$ is total. Here $u={ }_{\kappa} v$ yields $x \stackrel{(A 1)}{=} \rho_{u u}(x)=\rho_{v v}(y) \stackrel{(A 1)}{=} y$, whereas $u<_{\kappa} v$ and $v<_{\kappa} u$ yields $x \stackrel{(4.7)}{<} y$ and $y \stackrel{(4.7)}{<} x$, respectively.

- $<$ is transitive: Let $x \in X_{u}, y \in X_{v}, z \in X_{w}$, and assume $x<y<z$. From $x<y$ it follows that $\rho_{u v}(x) \leq_{u v} \rho_{u v}(y)$, hence by preservation of the ordering, $\rho_{u v w}(x) \leq_{u v w} \rho_{u v w}(y)$ holds. Analogously we obtain $\rho_{u v w}(y) \leq_{u v w} \rho_{u v w}(z)$, hence $\rho_{u v w}(x) \leq_{u v w} \rho_{u v w}(z)$ follows by the transitivity of $\leq_{u v w}$. Therefore either $\rho_{u v w}(x)<_{u v w} \rho_{u v w}(z)$ and we conclude $x<z$, or $\rho_{\text {uvw }}(x)=\rho_{\text {uvw }}(z)$. The latter implies $\rho_{\text {uvw }}(x)=$ $\rho_{u v w}(y)=\rho_{u v w}(z)$, and also $u<_{\kappa} v$ and $v<_{\kappa} w$. Therefore, by the transitivity of $<_{\kappa}, u<_{\kappa} w$ follows and thus $x<z$.

$(X, \cdot, t)$ is a commutative monoid.

- Commutativity of - is straightforward.

- Let $x \in X_{u}, y \in X_{v}, z \in X_{w}$. Then $(x y) z=\left(\rho_{u v}(x) \cdot u v \rho_{u v}(y)\right) z$, and the latest is equal to $\rho_{u v w}\left(\rho_{u v}(x) \cdot u v \rho_{u v}(y)\right) \cdot{ }_{u v w} \rho_{u v w}(z)$ since $\rho_{u v}(x) \cdot u v$ $\rho_{u v}(y) \in X_{u v}$. Since the $\rho$ 's preserve products and - is idempotent, the latest is equal to $\left(\rho_{u v w}(x) \cdot u v w \rho_{u v w}(y)\right) \cdot u v w \rho_{u v w}(z)$. Analogously follows that $x(y z)$ is equal to $\rho_{u v w}(x) \cdot u v w\left(\rho_{u v w}(y) \cdot{ }_{u v w} \rho_{u v w}(z)\right)$, and hence the associativity of $\cdot$ uvw implies the associativity of $\cdot$

- For $x \in X_{u}, t x \stackrel{(4.8)}{=} \rho_{u}(t) \cdot u \rho_{u}(x) \stackrel{(4.6)}{=} \rho_{u}(t) \cdot{ }_{u} x \stackrel{(4.2)}{=} u \cdot u x=x$ holds using that $u$ is the unit element of $\mathbf{X}_{u}$.

' is an order reversing bijection on $X$.

We start with two claims. 
- (C1) If $u<_{\kappa} v$ then for $x \in X_{u}, \rho_{v}\left(x^{\mu}\right)$ is the inverse of $\rho_{v}(x)$ in $\mathbf{X}_{v}$. Indeed, $\rho_{v}(x) \cdot v \rho_{v}\left(x^{\mu}\right) \stackrel{(4.6)}{=} \rho_{u \rightarrow v}(x) \cdot v \rho_{u \rightarrow v}\left(x^{\mu}\right) \stackrel{(4.2)}{=} \rho_{u \rightarrow v}\left(x \cdot u x^{\mu^{\mu}}\right)$. Note that $x \cdot{ }_{u} x^{\mu^{\mu}}=u^{\mu^{\mu}}$ holds not only if $u \in \kappa_{I}$ (see (4.1)), but also if $u \in \kappa_{O} \cup \kappa_{J}$, since due to the cancellativity of $\mathbf{X}_{u}, x \cdot{ }_{u} x^{\mu}=x \cdot u$ $\left(x \rightarrow{ }_{u} u^{\mu}\right) \stackrel{(3.4)}{=} x \cdot{ }_{u}\left(x^{-1_{u}} \cdot{ }_{u} u^{\mu^{\mu}}\right)=\left(x \cdot{ }_{u} x^{-1_{u}}\right) \cdot{ }_{u} u^{\mu^{\mu}}=u \cdot{ }_{u} u^{\mu^{\mu}}=u^{\mu}$. Therefore, $\rho_{u \rightarrow v}\left(x \cdot u x^{{ }^{u}}\right)=\rho_{u \rightarrow v}\left(u^{{ }^{u}}\right)$, which is equal to $\rho_{u \rightarrow v}(u) \stackrel{(4.2)}{=} v$ if $u \in \kappa_{o}$ (since then $u^{u}=u$ ), and is equal to $\stackrel{(A 2)}{=} \rho_{u \rightarrow v}(u) \stackrel{(4.2)}{=} v$ if $u \notin \kappa_{o}$. - (C2) If $u<_{\kappa} v$ then for $x \in X_{u}, \rho_{v}(x)^{{ }^{\nu}}=\rho_{v}\left(x^{{ }^{\mu}}\right)_{\downarrow_{v}}<\rho_{v}\left(x^{{ }^{\mu}}\right)$. First we prove $\rho_{v}(x)^{\nu} \cdot{ }_{v} \rho_{v}(x)=v_{\downarrow_{v}}$ : if $v \in \kappa_{J}$ then due to the cancellativity of $\mathbf{X}_{v}$ (see (4.1)), $\rho_{v}(x)^{\gamma} \cdot{ }_{v} \rho_{v}(x)=\rho_{v}(x) \cdot{ }_{v}\left(\rho_{v}(x) \rightarrow v v^{\nu}\right) \stackrel{(3.4)}{=} \rho_{v}(x) \cdot v$ $\left(\rho_{v}(x)^{-1} \cdot v_{v} v^{\gamma}\right)=\left(\rho_{v}(x) \cdot{ }_{v} \rho_{v}(x)^{-1}\right) \cdot v_{v} v^{\gamma}=v \cdot v v^{\gamma}=v^{\nu} \mathbf{X}_{v}$ is even, see (4.1) $v_{\downarrow_{v}}$, whereas if $v \in \kappa_{I}$ then $\rho_{v}(x)^{\nu} \cdot{ }_{v} \rho_{v}(x)=v^{\nu}=v_{\downarrow_{v}}$ holds by (4.1), and we are done. Now, multiplying both sides by $\rho_{v}\left(x^{{ }^{\mu}}\right)$ yields $\rho_{v}(x)^{\nu^{\prime}}=$ $v_{\downarrow_{v}} \cdot \rho_{v}\left(x^{\mu}\right)$ using (C1). If $v \in \kappa_{J}$ then by (4.1) we can apply claim (xiv) in Lemma 3.1 resulting in $\rho_{v}\left(x^{{ }^{u}}\right)_{\downarrow_{v}}=v_{\downarrow_{v}} \cdot{ }_{v} \rho_{v}\left(x^{{ }^{u}}\right)<v \cdot{ }_{v} \rho_{v}\left(x^{{ }^{\mu}}\right)=\rho_{v}\left(x^{{ }^{u}}\right)$, so we are done. If $v \in \kappa_{I}$ then by (4.1), $\mathbf{X}_{v}$ is an even involutive $\mathrm{FL}_{e^{-c h a i n}}$ with an idempotent falsum. Therefore, $\mathbf{X}_{v}=S p(\mathbf{A}, \mathbf{H})$ by Theorem 6.3, and since $\rho_{v}\left(x^{\mu}\right)$ is invertible by $(\mathrm{C} 1), \rho_{v}\left(x^{\mu}\right)$ is an element of $\mathbf{H}$ by claim (c) in Theorem 6.3. On the other hand, $v_{\downarrow}$ is clearly in $H^{\bullet}$. Therefore, $v_{\downarrow_{v}} \cdot v \rho_{v}\left(x^{\mu}\right) \stackrel{(6.8)}{=}\left(v \cdot v \rho_{v}\left(x^{\mu}\right)\right)_{\downarrow_{v}}=\rho_{v}\left(x^{\mu}\right)_{\downarrow_{v}} \stackrel{\rho_{v}\left(x^{\mu}\right) \in H}{<} \rho_{v}\left(x^{\mu}\right)$ holds.

- Since for $u \in \kappa,{ }^{\mu}$ is of order 2, so is ' over $X$ by (4.9), hence' is a bijection. It remains to prove that ' is order reversing. Let $X_{u} \ni x \leq$ $y \in X_{v}$. If $u=v$ then $x \leq_{u} y$ holds by (4.7), hence $y^{{ }^{\mu}} \leq_{u} x^{\mu^{u}}$ follows since $\mathbf{X}_{u}$ is involutive, thus $y^{\prime} \leq x^{\prime}$ holds by (4.7) and (A1). If $u<_{\kappa} v$ then $x \leq y$ implies $\rho_{v}(x) \leq_{u v} y$ by (4.7), hence $y^{\gamma} \leq_{v} \rho_{v}(x)^{\gamma}$ follows since $\mathbf{X}_{v}$ is involutive. Therefore, $y^{{ }^{\prime}} \leq_{v} \rho_{v}(x)^{\gamma} \stackrel{(C 2)}{<} \rho_{v}\left(x^{{ }^{\mu}}\right)$, and thus $y^{\gamma}<_{v} \rho_{v}\left(x^{\mu}\right)$ implies $y^{\gamma} \leq x^{\mu}$ by (4.7), yielding $y^{\prime} \leq x^{\prime}$ by (4.9). If $u>_{\kappa} v$ then $x \leq y$ implies $x<_{u} \rho_{u}(y)$ by (4.7), which is equivalent to $\rho_{u}(y)^{\mu^{\mu}}<_{u} x^{\mu^{\mu}}$ since $\mathbf{X}_{u}$ is involutive. By $(\mathrm{C} 2), \rho_{u}\left(y^{\gamma^{\prime}}\right) \leq_{u} x^{{ }^{\mu}}$ follows, which yields $y^{\nu} \leq x^{{ }^{\prime}}$ by (4.7), and hence $y^{\prime} \leq x^{\prime}$ follows by (4.9).

$t^{\prime}$ is a dualizing element of $(X, \leq$,$) .$ 
- It suffices to prove that for $x \in X$, there exists $x \rightarrow t^{\prime}$ and

$$
x \rightarrow t^{\prime}=x^{\prime},
$$

since the involutivity of ' then ensures $\left(x \rightarrow t^{\prime}\right) \rightarrow t^{\prime}=x$. Equivalently, that for $x, y \in X, x y \leq t^{\prime}$ if and only if $x \leq y^{\prime}$. Let $x \in X_{u}, y \in X_{v}$. Since $\cdot$ is commutative and since ' is an order reversing bijection, we may safely assume $u \leq_{\kappa} v$. Since $t^{\prime} \stackrel{(4.9)}{=} t^{\dagger} \in X_{t}$ and $t \leq_{\kappa} v$, by (4.8) $x y \leq t^{\dagger}$ is equivalent to $\rho_{v}(x) \cdot{ }_{v} \rho_{v}(y) \leq t^{\dagger}$. Since $\rho_{v}(x) \cdot{ }_{v} \rho_{v}(y) \in X_{v}$, $\rho_{v}(x) \cdot{ }_{v} \rho_{v}(y) \leq t^{\dagger}$ is equivalent to $\rho_{v}(x) \cdot{ }_{v} \rho_{v}(y) \leq_{v} v^{{ }^{\gamma}}$ : indeed, if $t=v$ then $\rho_{v}(x) \cdot_{v} \rho_{v}(y) \leq t^{\dagger}$ is equivalent to $\rho_{v}(x) \cdot_{v} \rho_{v}(y) \stackrel{(4.7)}{\leq}{ }_{v} t^{\dagger}=v^{\gamma}$, whereas if $t<_{\kappa} v$ then $v \notin \kappa_{O}$ and $\rho_{v}(x) \cdot_{v} \rho_{v}(y) \leq t^{\dagger}$ is equivalent to $\rho_{v}(x) \cdot_{v} \rho_{v}(y) \stackrel{(4.7)}{<_{v}} \rho_{v}\left(t^{\dagger}\right) \stackrel{(4.6)}{=} \rho_{t \rightarrow v}\left(t^{\dagger}\right) \stackrel{(A 2)}{=} \rho_{t \rightarrow v}(t) \stackrel{(4.2)}{=} v$, that is, equivalent to $\rho_{v}(x) \cdot v \rho_{v}(y) \stackrel{v \notin \kappa_{o},(4.1)}{\leq} v_{\downarrow_{v}} \stackrel{(4.1)}{=} v^{\gamma}$. Since $\mathbf{X}_{v}$ is residuated, $\rho_{v}(x) \cdot{ }_{v} \rho_{v}(y) \leq_{v} v^{\nu^{v}}$ is equivalent to $\rho_{v}(x) \leq_{v} y \rightarrow_{v} v^{\nu^{v}}=y^{\gamma^{v}}$, and by (4.7) it is equivalent to $x \leq y^{\gamma} \stackrel{(4.9)}{=} y^{\prime}$.

Summing up, we have shown that $(X, \leq)$ is a chain and $(X, \cdot, t)$ is a commutative monoid. Since $t^{\prime}$ is a dualizing element of $(X, \leq$, ), Lemma 3.3 shows that $(X, \leq$,$) is residuated and x \rightarrow y=\left(x y^{\prime}\right)^{\prime}$. Since' ${ }^{\prime}$ (given in (4.9)) coincides with the residual complement of $\mathbf{X}$ (given by $x \rightarrow t^{\prime}$, see (4.17)), and since' ${ }^{\prime}$ is an order reserving involution on $X$, it follows that $\mathbf{X}$ is involutive. Finally, by (4.1), $\mathbf{X}_{t}$ and hence also $\mathbf{X}$ is odd if $t \in \kappa_{o}, \mathbf{X}$ is even with a non-idempotent falsum if $t \in \kappa_{J}$, and $\mathbf{X}$ is even with an idempotent falsum if $t \in \kappa_{I}$.

(3): Let $\mathcal{A}=\left\langle\mathbf{X}_{u}, \rho_{u \rightarrow v}\right\rangle_{\kappa}$ be a bunch of layer algebras, and adapt the notations in Definition 4.1 and the definitions in claim (2). To see that the universe of the $u^{\text {th }}$ layer algebra of $\mathcal{A}_{\left(\mathcal{X}_{\mathcal{A}}\right)}$ is equal to $X_{u}$ which is the universe of the $u^{\text {th }}$ layer algebra $\mathbf{X}_{u}$ of $\mathcal{A}$, we need to prove that for $x \in X$ (where $X$ is given in (4.5)), $x \rightarrow x=u$ if and only if $x$ is in $X_{u}$, the universe of the $u^{\text {th }}$-layer algebra: $x \in X$ implies that $x \in X_{v}$ for some

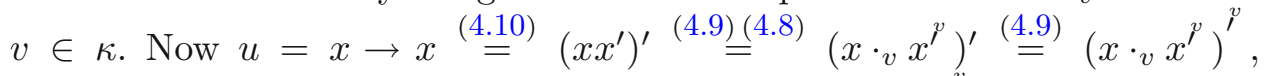
where the last equality holds since $X_{v}$ is closed under ${ }^{\nu}$ and ${ }_{v}$ and thus $x \cdot{ }_{v} x^{y}$ is in $X_{v}$. Therefore, $u \in X_{v}$ follows. Hence $u=v$ must hold since $u \in X_{u}$ and $X$ is the disjoint union of the $X_{u}$ 's by (4.5). The definition of the ordering relation in claim (1) and (4.7) show that the ordering of the $u^{\text {th }}$ layer algebra of $\mathcal{A}_{\left(\mathcal{X}_{\mathcal{A}}\right)}$ is the same as the ordering $\leq_{u}$ of the $u^{\text {th }}$ layer algebra $\mathbf{X}_{u}$ of $\mathcal{A}$. Likewise show (4.8) and the definition of the monoidal 
operation in claim (1) that the monoidal operation of the $u^{\text {th }}$ layer algebra of $\mathcal{A}_{\left(\mathcal{X}_{\mathcal{A}}\right)}$ is the same as the monoidal operation ${ }^{u}$ of the $u^{\text {th }}$ layer algebra $\mathbf{X}_{u}$ of $\mathcal{A}$. Since both the $u^{\text {th }}$ layer algebra of $\mathcal{A}_{\left(\mathcal{X}_{\mathcal{A}}\right)}$ and the $u^{\text {th }}$ layer algebra $\mathbf{X}_{u}$ of $\mathcal{A}$ are involutive $\mathrm{FL}_{e}$-chains over the same universe, equipped with the same ordering relation and the same product operation, their residual operations - which are uniquely determined by these - must coincide, too. The unit element of $\mathbf{X}_{u}$ is $u$, therefore $u$ acts as the unit element of the $u^{\text {th }}$ layer algebra of $\mathcal{A}_{\left(\mathcal{X}_{\mathcal{A}}\right)}$ (which is over the same set $X_{u}$ and is equipped with the same monoidal operation, as we have seen above). Finally, the falsum constant of $\mathbf{X}_{u}$ is $u^{\mu^{\mu}}$. On the other hand, the falsum constant of the $u^{\text {th }}$ layer algebra of $\mathcal{A}_{\left(\mathcal{X}_{\mathcal{A}}\right)}$ is $u \rightarrow u^{\prime} \stackrel{(4.9)}{=} u \rightarrow u^{{ }^{u}}=u \rightarrow{ }_{u} u^{{ }^{u}}=u^{{ }^{u}}$, where the last equality holds by residuation since $u$ is the unit element over $X_{u}$. Summing up, $\mathcal{A}_{\left(\mathcal{X}_{\mathcal{A}}\right)}=\mathcal{A}$.

Let $\mathbf{X}=(X, \leq, \cdot, \rightarrow, t, f)$ be an odd or even involutive $\mathrm{FL}_{e}$-chain, and adapt the definitions in claim (1). We have seen in the proof of claim (1) that the $X_{u}$ 's are nonempty. It is straightforward that they are disjoint, too, and their union, which is the universe of $\mathcal{X}_{\mathcal{A}_{\mathbf{X}}}$, see (4.5), is equal to $X$. To prove that the ordering of $\mathbf{X}$ and of $\mathcal{X}_{\mathcal{A}_{\mathbf{X}}}$ coincide, first we prove the following statement. If $v<u$ and $x \in X_{v}$ then

$$
\rho_{u}(x)=\min \left\{z \in X_{u}: z \geq x\right\}>x .
$$

Indeed, $u x \in X_{u}$ by claim (xvi) in Lemma 3.1, and $u x \geq x$ holds since $u x \geq t x=x$. By contradiction, assume that there exists $z \in X_{u}$ such that $x<z<u x$. Since $\leq$ is total, by adjointness $z^{\prime \prime}<u x$ is equivalent to $f<z^{\prime}(u x)=\left(z^{\prime} u\right) x \stackrel{z^{\prime} \in X_{u}}{=} z^{\prime} x$. Finally, since $\leq$ is total, by adjointness $f<z^{\prime} x$ is equivalent to $x>z^{\prime \prime}=z$, a contradiction. Referring to (4.18) a moment's reflection shows that the ordering $\leq$ of $X$ coincides with the ordering of $\mathcal{X}_{\mathcal{A}_{\mathbf{X}}}$ given by (4.7). Since for $x \in X_{u}$ and $y \in X_{v}$, $x y \stackrel{L 3.1(v)}{=}(u u x)(v v y)=((u v) x)((u v) y)=\rho_{u v}(x) \rho_{u v}(y)=\rho_{u v}(x) \cdot u v \rho_{u v}(y)$, the monoidal operation - (of $X$ ) coincides with $\cdot$ given in (4.8). Since both $\mathbf{X}$ and $\mathcal{X}_{\mathcal{A}_{\mathbf{x}}}$ are involutive $\mathrm{FL}_{e}$-chains over the same universe, equipped with the same ordering relation and the same product operation, their residual operations - which are uniquely determined by these - must coincide, too. By (4.3), the unit element of $\mathbf{X}_{t}$ is $t$, and hence the unit element of $\mathcal{X}_{\mathcal{A}_{\mathbf{X}}}$ is also $t$. Finally, the falsum constant of $\mathbf{X}$ is $f$, hence the falsum constant of $\mathbf{X}_{t}$ is $t^{\dagger}=t \rightarrow t^{\prime} \stackrel{L 3.1(i)}{=} t \rightarrow f \stackrel{L 3.1(i)}{=} f$. Therefore, the falsum constant of $\mathcal{X}_{\mathcal{A}_{\mathbf{X}}}$ is also $t^{\prime}=f$. Summing up, $\mathcal{X}_{\mathcal{A}_{\mathbf{X}}}=\mathbf{X}$. 
Remark 4.3. Two elements of the construction of $\mathcal{X}_{\mathcal{A}}$ in Lemma $4.2 /(2)$ are reminiscent to the Płonka sum construction [48]. In the Płonka sum construction the universe of the algebra is the disjoint union of the universes of the algebras in the direct system.So is in our construction, c.f. (4.5). In the Płonka sum construction every binary operator $\circ \in\{\cdot, \rightarrow, \wedge, \vee\}$ would be given in terms of the direct system as follows: for $x \in X_{u}, y \in X_{v}$,

$$
x \circ y=\rho_{u \rightarrow u v}(x) \circ_{u v} \rho_{u \rightarrow u v}(y)
$$

In our construction it is the case only for $\circ \in\{\cdot\}$, c.f. (4.8). As for $\circ \in\{\rightarrow\}$, if $\rightarrow$ were given by (4.19) then for any positive idempotent elements $u<v$, it would hold true that $v \rightarrow u=u \rightarrow v$ since $v \rightarrow u \stackrel{(4.19)}{=} v \rightarrow{ }_{v} v \stackrel{(4.19)}{=}$ $u \rightarrow v$. But in an involutive $\mathrm{FL}_{e}$-chain it would be equivalent to $v u^{\prime}=$ $u v^{\prime}$ by Lemma $3.1 /$ (ii), a contradiction, since $v u^{\prime}=v$ and $u v^{\prime}=v^{\prime}$ (as shown by $v \stackrel{\text { L3.1(vi) }}{=} \tau(v) \stackrel{\text { L3.1(ii) }}{=}\left(v v^{\prime}\right)^{\prime} \stackrel{\text { L3.1(iv) }}{\leq} v u^{\prime} \leq v t=v$ and $v^{\prime}=t v^{\prime} \leq$ $u v^{\prime} \leq v v^{\prime} \stackrel{L 3.1(i i)}{=} \tau(v)^{\prime} \stackrel{L 3.1(v i)}{=} v^{\prime}$, respectively). Finally, notice that Płonka's definition doesn't work for $\circ \in\{\wedge, \vee\}$ since (4.19) would render different elements of the universe equal. Indeed, if $u<v$ are positive idempotent elements, $x \in X_{u}$ and $\rho_{u \rightarrow v}(x)=y \in X_{v}$ then according to (4.19), $x \wedge y=$ $\rho_{u \rightarrow v}(x) \wedge_{v} y=y=\rho_{u \rightarrow v}(x) \vee_{v} y=x \vee y$ yielding $x=y$, a contradiction since $x$ and $y$ are in different layers. Even though our definition is applied in our specific (residuated lattice) setting only, we have introduced in (4.7) a general way of ordering Ptonka sums, call it the directed lexicographic order. Making use of the (slight) similarity between our construction and that of Płonka's would have made the related proof only a few lines shorter. Therefore, we have included those few lines, too, to make the treatment self-contained.

Bunches of layer algebras will, in turn, be represented by bunches of layer groups in Section 7.1. To that end, in the following two sections the necessary auxiliary results (along with some which are more general than what we actually need in this paper) will be developed.

\section{Even Involutive $\mathbf{F L}_{e}$-Chains with Non-idempotent Falsum Constants - Changing the Falsum Constant}

An abelian o-group is called discrete, if there exists the smallest positive element greater than the unit element. It is equivalent to saying that the abelian o-group is discretely ordered, or that its induced cancellative odd involutive $\mathrm{FL}_{e}$-algebra is discretely ordered. In the following definition the 
residuated chain reduct of the $\mathrm{FL}_{e}$-chain is left unchanged, and only the falsum constant, and thus also the residual complement are changed slightly.

Definition 5.1. For a discretely ordered, cancellative, odd, involutive $\mathrm{FL}_{e^{-}}$ chain $\mathbf{X}=(X, \leq, \cdot, \rightarrow, t, t)$, let

$$
\mathbf{X}_{\downarrow}=\left(X, \leq, \rightarrow, t, t_{\downarrow}\right) .
$$

For a discretely ordered, cancellative, even, involutive $\mathrm{FL}_{e}$-chain $\mathbf{Y}=(Y, \leq$ $\left., \cdot, \rightarrow, t, t_{\downarrow}\right)$, let

$$
\mathbf{Y}_{\uparrow}=(Y, \leq, \cdot, \rightarrow, t, t) .
$$

Lemma 5.2. Let $\mathbf{X}$ and $\mathbf{Y}$ be discretely ordered, cancellative, involutive $F L_{e}$-chains, $\mathbf{X}$ is odd, $\mathbf{Y}$ is even. Then

(1) $\mathbf{X}_{\downarrow}$ is a discretely ordered, cancellative, even, involutive $F L_{e}$-chain,

(2) $\mathbf{Y}_{\uparrow}$ is a discretely ordered, cancellative, odd, involutive $F L_{e}$-chain,

(3) $\mathbf{X}_{\downarrow \uparrow}=\mathbf{X}$ and $\mathbf{Y}_{\uparrow \downarrow}=\mathbf{Y}$.

Proof. The rest being obvious we prove the involutivity of $\mathbf{X}_{\downarrow}$ and $\mathbf{Y}_{\uparrow}$. (1): To see that $\mathbf{X}_{\downarrow}$ is involutive, denote the residual complement operation of $\mathbf{X}$ by ', and the inverse operation of $\lambda(\mathbf{X})$ by ${ }^{-1}$. Then $x \rightarrow t_{\downarrow} \stackrel{(3.4)}{=}$ $x^{-1} t_{\downarrow} \stackrel{(3.3)}{=} x^{\prime} t_{\downarrow} \stackrel{\text { L3.1(xiv) }}{=}\left(x^{\prime}\right)_{\downarrow}$, and hence (3.1) confirms involutivity. (2): To see that $\mathbf{Y}_{\uparrow}$ is involutive, denote the residual complement operation of $\mathbf{Y}$ by $^{i}$. Then $y \rightarrow t \stackrel{L 3.1(i i)}{=}\left(y t^{\boldsymbol{i}}\right)^{i} \stackrel{\text { L3.1(i) }}{=}(y f)^{\boldsymbol{i}} \stackrel{\mathbf{Y}}{\text { is even }}\left(y t_{\downarrow}\right)^{\boldsymbol{i}} \stackrel{\text { L3.1(xiv) }}{=} y_{\downarrow}{ }^{\boldsymbol{\prime}}$ holds, and hence (3.1) confirms involutivity.

\section{Even Involutive $\mathbf{F L}_{e}$-Algebras with Idempotent Falsum Constants - Subgroup Splits of Odd Involutive $\mathbf{F L}_{e}$-Algebras}

As an investigation into the structure of residuated lattices which are not necessarily totally ordered, a one-to-one correspondence between pairs of an odd involutive $\mathrm{FL}_{e}$-algebra and a cancellative subalgebra of it, and even involutive $\mathrm{FL}_{e}$-algebras where the residual complement of the unit element is idempotent will be proved in this section. In groups the unit element has two different roles to play. It serves as the unit element of the multiplication, and also the product of any element by its inverse is equal to it. We shall replace the unit element of any odd involutive $\mathrm{FL}_{e}$-algebra by two elements, 
each will inherit a single role. This way both the unit element itself and its two roles will be "split" into two. Some anticipatory examples: the unit-split algebra of the one-element group will be the two-element Boolean algebra, and the unit-split algebra of the Sugihara lattice $\mathbf{S}_{\mathbb{Z}}$ will be the Sugihara lattice $\mathbf{S}_{\mathbb{Z}^{\star}}$, (both named by Meyer, see [2][p. 414]). Moreover, not only the unit element, but in fact any subgroup of an odd involutive $\mathrm{FL}_{e}$-algebra can be "split", that is, each element of the subgroup of an odd involutive $\mathrm{FL}_{e^{-}}$ algebra will be replaced by two elements. We prove that by splitting (and thus "doubling") a subgroup of any odd involutive $\mathrm{FL}_{e}$-algebra we obtain an even involutive $\mathrm{FL}_{e}$-algebra with an idempotent falsum constant, and each even involutive $\mathrm{FL}_{e}$-algebra with an idempotent falsum constant arises this way in a unique manner (Theorem 6.3).

Such a splitting method has roots in the literature. In [11] an analogous idea has been applied to abelian pregroups. Our method here is both more general and more specific: the algebras that we split are more general than that of [11], however, in our approach the (second) algebra we use in the splitting procedure is the two element Boolean algebra (each element is split into two elements only) whereas in [11] it is taken from the more general class of zero-pregroups. The notion of the melting structure (of a pregroup) in [11] is analogous to the image (of the even involutive $\mathrm{FL}_{e}$-algebra with idempotent falsum constant) under what we call its canonical homomorphism. The splitting procedure in [11] is precursor also for the partial lex-product construction of [31], which when further generalized to partial sublex-products has been capable to describe the structure of all odd, involutive $\mathrm{FL}_{e}$-chains which have only finitely many idempotent elements [31,32].

Definition 6.1. Let $\mathbf{Y}=\left(Y, \wedge, \vee, \star, \rightarrow_{\star}, t, f\right)$ be an even $\mathrm{FL}_{e^{-}}$algebra with an idempotent falsum constant. Denote its residual complement by ${ }^{\star}$. Let

$$
\pi_{1}(\mathbf{Y})=\mathbf{X}=(X, \dot{\wedge}, \dot{V}, \cdot, \rightarrow, t, t) \operatorname{and} \pi_{2}(\mathbf{Y})=\mathbf{H}=(H, \dot{\wedge}, \dot{\vee}, \cdot, \rightarrow, t, t)
$$

be given by

$$
H=\{x \in Y: x \star f<x\}, H^{\bullet}=\{x \star f: x \in H\}, X=Y \backslash H^{\bullet},
$$

for $y \in Y$,

$$
h_{\mathbf{Y}}(x)= \begin{cases}x & \text { if } x \in X \\ x_{\uparrow} & \text { if } x \in H^{\bullet}\end{cases}
$$


and for $x, y \in X$,

$$
\begin{aligned}
x \dot{\wedge} y & =h_{\mathbf{Y}}(x \wedge y), \\
x \dot{\vee} y & =h_{\mathbf{Y}}(x \vee y), \\
x y & =h_{\mathbf{Y}}(x \star y), \\
x \rightarrow y & =h_{\mathbf{Y}}\left(x \rightarrow_{\star} y\right) .
\end{aligned}
$$

Definition 6.2. Let $\mathbf{X}=(X, \wedge, \vee, \cdot, \rightarrow, t, t)$ be an odd involutive $\mathrm{FL}_{e^{-}}$ algebra with residual complement '. Let $\mathbf{H} \leq \mathbf{X}$ (over $H \subseteq X$ ), $\mathbf{H}$ cancellative $^{15}$. Let $S p(\mathbf{X}, \mathbf{H})$, the $\mathbf{H}$-split of $\mathbf{X}$ be

$$
\mathbf{Y}=\left(Y, \wedge_{Y}, \vee_{Y}, \star, \rightarrow_{\star}, t, t^{\bullet}\right),
$$

where $H^{\bullet}=\left\{h^{\bullet}: h \in H\right\}$ is a copy of $H$ disjoint from $X$,

$$
Y=X \cup H^{\bullet},
$$

the lattice ordering $\leq$ of $X$ is extended to $Y$ by letting

$$
a^{\bullet}<_{Y} b^{\bullet} \text { and } x<_{Y} a^{\bullet}<_{Y} y \text { for } a, b \in H, a<b, x, y \in X, x<a \leq y,
$$
$h: Y \rightarrow X$,

$$
h(x)=\left\{\begin{array}{lll}
x & \text { if } & x \in X \\
x_{\uparrow} & \text { if } & x \in H^{\bullet}
\end{array},\right.
$$

where $\uparrow$ and $\downarrow$ denote the neighbour operations of $\mathbf{Y}$,

$$
x \star y=\left\{\begin{array}{ll}
h(x) h(y) & \text { if } h(x) h(y) \notin H \text { or } x, y \in H \\
(h(x) h(y))_{\downarrow} & \text { if } \neg(x, y \in H) \text { and } h(x) h(y) \in H
\end{array},\right.
$$

$\stackrel{\star}{\star}: Y \rightarrow Y$,

$$
\begin{aligned}
x^{\dagger} & =\left\{\begin{array}{lll}
x^{\prime} & \text { if } & x \in X \backslash H \\
\left(x^{\prime}\right)_{\downarrow} & \text { if } & x \in H \\
\left(x_{\uparrow}\right)^{\prime} & \text { if } & x \in H^{\bullet}
\end{array},\right. \\
x \rightarrow \star y & =\left(x \star y^{\dagger}\right)^{\dagger} .
\end{aligned}
$$

TheOREM 6.3. The following statements hold true.

(1) Let $\mathbf{X}$ be an odd involutive $F L_{e}$-algebra, $\mathbf{H}$ be a cancellative subalgebra of it, and $\mathbf{Y}$ be $S p(\mathbf{X}, \mathbf{H})$, the $\mathbf{H}$-split of $\mathbf{X}$. Then

(a) $\mathbf{Y}$ is an even $F L_{e}$-algebra with an idempotent falsum constant.

(b) If $\mathbf{X}$ is cancellative then for $x \in Y, x \star x^{{ }^{\star}}=t^{\bullet}$ holds.

\footnotetext{
${ }^{15}$ Equivalently, $\lambda(\mathbf{H})$ is a lattice ordered abelian group by Lemma 3.2.
} 
(c) $\{x \in S p(\mathbf{X}, \mathbf{H}): x$ is invertible in $S p(\mathbf{X}, \mathbf{H})\}=\mathbf{H}$.

(2) Let $\mathbf{Y}$ be an even involutive $F L_{e}$-algebra with an idempotent falsum constant. Then

(a) there exists a unique pair $\langle\mathbf{X}, \mathbf{H}\rangle$ of an odd involutive $F L_{e}$-algebra $\mathbf{X}=(X, \dot{\wedge}, \dot{V}, \cdot, \rightarrow, t, t)$ and a cancellative subalgebra $\mathbf{H}$ of $\mathbf{X}$ such that $\mathbf{Y}$ is $S p(\mathbf{X}, \mathbf{H})$, the $\mathbf{H}$-split of $\mathbf{X} . \mathbf{X}$ and $\mathbf{H}$ are given by $\pi_{1}(\mathbf{Y})$ and $\pi_{2}(\mathbf{Y})$, respectively.

(b) If for $x \in Y, x \star x^{t^{+}}=f$ holds then $\mathbf{X}$ is cancellative.

(c) $h_{\mathbf{Y}}$ is a surjective homomorphism (called the canonical homomorphism of $\mathbf{Y}$ ) from $\mathbf{Y}$ onto $\mathbf{X}$, that is, it holds true that

$$
\mathbf{X}=h_{\mathbf{Y}}(S p(\mathbf{X}, \mathbf{H})) .
$$

Proof. (a): It is obvious from (6.6) that

$$
\text { for } a \in H, a \text { is the unique cover of } a^{\bullet} \text {. }
$$

Therefore, by letting $k: X \rightarrow Y$,

$$
k(x)=\left\{\begin{array}{lll}
x & \text { if } & x \in X \backslash H \\
x & \text { if } & x \in H
\end{array},\right.
$$

a moment's reflection shows that $\leq_{Y}$ is a lattice ordering on $Y$ and the corresponding lattice operations are given by

$$
\begin{aligned}
& x \wedge_{Y} y=y \wedge_{Y} x=\left\{\begin{array}{ll}
y & \text { if } h(y) \leq h(x), y \in H^{\bullet} \\
h(x) \wedge h(y) & \text { otherwise }
\end{array},\right. \\
& x \vee_{Y} y=y \vee_{Y} x=\left\{\begin{array}{lll}
h(x) \vee h(y) & \text { if } & h(x) \vee h(y) \notin H \\
k(h(x) \vee h(y)) & \text { if } & \{h(x), h(y)\} \not \supset h(x) \vee h(y) \in H \\
h(x) \vee h(y) & \text { if } & h(x) \leq h(y), y \in H \\
k(h(x) \vee h(y)) & \text { if } & h(x) \leq h(y), y \in H^{\bullet}
\end{array} .\right.
\end{aligned}
$$

It is straightforward from (6.8) that $\star$ is commutative. $t$ is the unit element of $\star$ : using that $t$ is the unit element over $X$,

$$
x \star t \stackrel{(6.8),(6.7)}{(6.7)}=\left\{\begin{array}{lll}
h(x) t=h(x)=x & \text { if } \quad h(x) \notin H \text { or } x \in H \\
(h(x) t)_{\downarrow}=h(x)_{\downarrow}=x & \text { if } \quad x \notin H \text { and } h(x) \in H
\end{array} .\right.
$$

We obtain $a^{\bullet}=a_{\downarrow}<a$ for $a \in H$ from (6.10), therefore, ${ }^{\star}$ in (6.9) is clearly an order reversing involution by (3.1).

As for the associativity of $\star$, first notice that

$$
x \star y \in H \text { if and only if } x, y \in H .
$$


Indeed, if $x, y \in H$ then $x \star y \stackrel{(6.8)}{=} h(x) h(y) \stackrel{(6.7)}{=} x y \in H$ since $H$ is closed under $\cdot$. If $\neg(x, y \in H)$ then either $h(x) h(y) \in H$ in which case $x \star y \stackrel{(6.8)}{\in} H^{\bullet}$ implies $x \star y \notin H$, or $h(x) h(y) \notin H$ in which case $x \star y \stackrel{(6.8)}{=} h(x) h(y) \notin H$, hence we are done. Next notice that

$$
\text { for } x, y \in Y, h(x \star y)=h(x) h(y) \text {. }
$$

Indeed, if the first row of (6.8) defines the value of $x \star y$ then $x \star y=$ $(h(x) h(y))_{\downarrow}$ and $h(x) h(y) \in H$, and hence $h(x \star y)=h\left((h(x) h(y))_{\downarrow}\right) \stackrel{(6.7)}{=}$ $(h(x) h(y))_{\downarrow \uparrow}=h(x) h(y)$, whereas if $x \star y \stackrel{(6.8)}{=} h(x) h(y)$ then $h(x \star y)=$ $h(h(x) h(y))=h(x) h(y)$ since $h$ maps to $X, X$ is closed under $\cdot$, and $h$ is the identity on $X$.

Now, $h((x \star y) \star z)=h(x \star(y \star z))$ readily follows from (6.12) and the associativity of $\cdot$. Therefore, by $(6.7),(x \star y) \star z \neq x \star(y \star z)$ can only be possible if one side is in $H$ and the other side is in $H^{\bullet}$. However, (6.11) shows that if one side is in $H$ then $x, y, z \in H$, and hence, since $H$ is closed under $\star$ by (6.11), also the other side must be in $H$.

It is easily seen that

$$
\star \text { is isotone with respect to } \leq_{Y} \text {. }
$$

Indeed, let $x, y, z \in Y$ with $y<_{Y} z$. Since $h$ and $\cdot$ are increasing with respect to $\leq$, it follows that $h(x) h(y) \leq h(x) h(z)$. Now by $(6.8), x \star y \leq_{Y} x \star z$ clearly holds if $h(x) h(y)<h(x) h(z)$, hence we may assume $h(x) h(y)=h(x) h(z)$. But then the only way for $x \star y \mathbb{Z}_{Y} x \star z$ to hold is if $x \star y=h(x) h(y)$ and $x \star z=(h(x) h(z))_{\downarrow}$, which by (6.8) leads to assuming $\neg(x, z \in H)$, $h(x) h(z) \in H$ and either $h(x) h(y) \notin H$ or $x, y \in H$. It follows that $x, y \in H$. But then $h(x) h(y)=x y<x h(z) \leq h(x) h(z)$ is a contradiction, where the strict inequality follows from $y<h(z)$ and since $x$ has an inverse.

To prove that $\star$ is residuated, and that $x \rightarrow_{\star} y=\left(x \star y^{\prime^{*}}\right)^{\prime^{\prime}}$, by Lemma 3.3 it suffices to verify that $t^{\bullet}$ is a dualizing element of $(Y, \leq, \star)$. Since ${ }^{\star}$ is clearly an order reversing involution, it suffices to verify that that $x \rightarrow_{\star} t_{\downarrow}$ exists and is equal to $x^{{ }^{\star}}$. It amounts to verifying only three cases. (i) If $x \in H$ then $x^{\star^{\prime}}=\left(x^{\prime}\right)_{\downarrow} \in H^{\bullet}$ and $x \star x^{\star} \stackrel{(6.9)}{=} x \star\left(x^{\prime}\right)_{\downarrow} \stackrel{(6.8)}{=}\left(x x^{\prime}\right)_{\downarrow}=t_{\downarrow}$, where in the last equality we used that $\mathbf{H}$ is a subgroup, hence $x^{\prime}$ is the inverse of $x$ in $\mathbf{X}$. On the other hand, for $z>x^{t^{*}}$ it follows that $z \geq x^{\prime}$ and hence

$x \star z \stackrel{(6.13)}{\geq} x \star x^{\prime} \stackrel{x^{\prime} \in H_{,}(6.8)}{=} x x^{\prime}=t>t_{\downarrow}$. Therefore, by residuation, $x \rightarrow_{\star} t_{\downarrow}$ exists and is equal to $x^{{ }^{\star}}$. (ii) If $x \in H^{\bullet}$ then $x=y_{\downarrow}$ for some $y \in H$ and 
$x^{\star}=y^{\prime}=\left(x_{\uparrow}\right)^{\prime} \stackrel{(3.1)}{=} x^{\prime} \downarrow>x^{\prime}$. We obtain $x \star x^{\star}=y_{\downarrow} \star y^{\prime} \stackrel{(6.8)}{=}\left(y y^{\prime}\right)_{\downarrow}=t_{\downarrow}$.

On the other hand, for $z>x^{t^{*}}$ it follows that $z \geq x^{\prime}$ and hence $x \star z \stackrel{(6.13)}{\geq}$ $y_{\downarrow} \star x^{\prime} \stackrel{(6.8)}{=}\left(y x^{\prime}\right)_{\downarrow}=\left(y y^{\prime} \uparrow\right)_{\downarrow}$. Here $y y^{\prime} \uparrow>t$ holds by residuation since $y^{\prime} \uparrow>y^{\prime}$, hence $x \star z>t_{\downarrow}$ follows. (iii) Finally, if $x \in X \backslash H$ then $x^{\prime^{\star}}=x^{\prime} \in X \backslash H$. The case $x \star x^{t^{*}}=x \star x^{\prime} \in H$ leads to $x \star x^{\star}=x \star x^{\prime} \stackrel{(6.8)}{=}\left(x x^{\prime}\right)_{\downarrow} \leq t_{\downarrow}$, whereas if $x \star x^{\dagger}=x \star x^{\prime} \in X \backslash H$ then $x \star x^{\star}=x \star x^{\prime} \stackrel{(6.8)}{=} x x^{\prime} \leq t$, but due to $x \star x^{\dagger} \in X \backslash H$ and $t \in H$ equality cannot hold, hence here too, $x \star x^{\star} \leq t_{\downarrow}$ follows. On the other hand if $z>x^{{ }^{\prime}}=x^{\prime}$ then $x \star z \geq(x z)_{\downarrow}>t_{\downarrow}$ holds by (6.8) and by residuation, respectively.

Summing up, the falsum-free reduct of $\mathbf{Y}$ is an involutive commutative residuated lattice with residual complement operation ${ }^{t}$. By the second row of (6.9), $t^{\dagger}=\left(t^{\prime}\right)_{\downarrow}=t_{\downarrow}$, and $t_{\downarrow}$ is idempotent by the second row of (6.8). A particular instance of (6.10) shows that $t$ is the unique cover of $t^{\bullet}$, hence $t^{\bullet}=t_{\downarrow}$ and thus $\mathbf{Y}$ is even.

(b): Since

$$
h(x) h\left(x^{\prime}\right)=x x^{\prime} \stackrel{L 3.1(x)}{=} f=t \in H,
$$

for $x \in Y, x \star x^{\dagger} \stackrel{(6.9)}{=}$

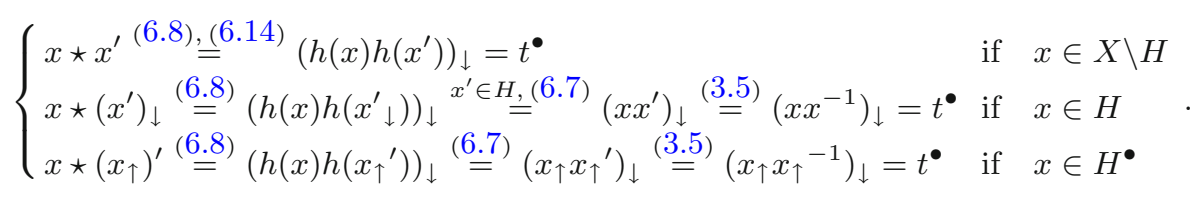

(c): Since $t \in H$ and $H^{\bullet}$ is disjoint from $H$, referring to (6.7), it follows from (6.8) that $x \star y$ can be equal to $t$ only if $x, y \in H$. On the other hand, every element of $H$ is invertible in $\mathbf{X}$ by claim (xi) in Lemma 3.1, since $\mathbf{H}$ is cancellative by assumption. Hence $x \star x^{-1} \stackrel{(6.8)}{=} h(x) h\left(x^{-1}\right) \stackrel{(6.7)}{=} x x^{-1}=t$.

(a): Unicity of $\mathbf{H}$ follows from claim (c), and it readily implies the unicity of $\mathbf{X}$, too, by (6.5). Let $\mathbf{X}=\pi_{1}(\mathbf{Y})$ and $\mathbf{H}=\pi_{2}(\mathbf{Y})$. Denote $x \bullet=x \star f$ for $x \in H$.

(i) Any element $x$ of $H$ is invertible, that is, $x \star x^{\dagger}=t$ holds, where ${ }^{\dagger}$ is given by

$$
x^{\dagger}=x \rightarrow \star t .
$$

Indeed, $t \geq x \star\left(x \rightarrow_{\star} t\right) \stackrel{L 3.1(i i)}{=} x \star\left(x \star t^{\dagger}\right)^{\dagger^{\star}} \stackrel{L 3.1(i)}{=} x \star(x \star f)^{\star} \not \leq f$, where the latest step holds by residuation since $x \in H$, that is $x \star f<$ 
Table 3. Product Table

\begin{tabular}{llll}
\hline$\star$ & $y \in X \backslash H$ & $l \in H$ & $l^{\bullet} \in H^{\bullet}$ \\
\hline$x \in X \backslash H$ & $\in(X \backslash H) \cup H^{\bullet}$ & $\in X \backslash H$ & $x \star l \in X \backslash H$ \\
$m \in H$ & $\in(X \backslash H)$ & $\in H$ & $(m \star l)^{\bullet} \in H^{\bullet}$ \\
$m^{\bullet} \in H^{\bullet}$ & $m \star y \in X \backslash H$ & $(m \star l)^{\bullet} \in H^{\bullet}$ & $(m \star l)^{\bullet} \in H^{\bullet}$
\end{tabular}

$x$, and it implies $(x \star f)^{t^{\dagger}}>x^{\dagger}$. Since $\mathbf{Y}$ is even, $t \geq x \star x^{\dagger} \not \leq f$ implies $x \star x^{\dagger}=t$.

(ii) $H \cap H^{\bullet}=\emptyset$. Indeed, if $x \in H^{\bullet}$, that is, if $x=y \star f$ for some $y \in H$ then $x \star f=(y \star f) \star f=y \star(f \star f)=y \star f=x$ ensures $x \notin H$.

(iii) $t \in H \subseteq X$. Indeed, $t \in H$ because of $t \star f=f<t$, and $H \subseteq X$ readily follows from (6.1) and claim (ii). Hence it holds true that

$$
Y=(X \backslash H) \dot{\cup} H \dot{\cup} H^{\bullet} .
$$

(iv) Next we prove $m^{\bullet}=m_{\downarrow}<m$ for $m \in H$. Indeed, the assumption $m \star f<z<m$ would yield $f=t \star f \stackrel{(i)}{=}\left(m^{\dagger} \star m\right) \star f=m^{\dagger} \star$ $(m \star f) \stackrel{\text { L3.1(viii) }}{<} m^{\dagger} \star z \stackrel{\text { L3.1(viii) }}{<} m^{\dagger} \star m=t$, a contradiction to $t$ covering $f$.

(v) For $m \in H$ and $y \in(X \backslash H) \cup H^{\bullet}$ it holds true that

$$
m \bullet \star y=m \star y .
$$

Indeed, $(X \backslash H) \cup H^{\bullet}=Y \backslash H$ holds by (6.15), therefore $y \star f=y$. We obtain $m \star y=m \star(y \star f)=(m \star f) \star y=m^{\bullet} \star y$, as stated.

(vi) For $m \in H, m^{\dagger} \in H: m^{\dagger} \notin H$ would imply $t \stackrel{(i)}{=} m \star m^{\dagger} \stackrel{(v)}{=}$ $m \bullet \star m^{\dagger}=(m \star f) \star m^{\dagger}=\left(m \star m^{\dagger}\right) \star f \stackrel{(i)}{=} t \star f=f$, a contradiction.

The following product table holds true ${ }^{16}$, see Table 3 .

$\star_{(2,2)}$ : Since $m$ is invertible by claim (i), $(m \star l) \star f=m \star(l \star f)<m \star l$ follows by claim (viii) in Lemma 3.1.

$$
\begin{aligned}
\star_{(2,3)}: & m \star l \cdot=m \star(l \star f)=(m \star l) \star f \stackrel{{ }_{(2,2)}}{=}(m \star l) \bullet \in H^{\bullet} . \\
\star_{(3,3)}: & m \bullet \star l \bullet=(m \star f) \star(l \star f)=(m \star l) \star(f \star f)=(m \star l) \star f \stackrel{\star(2,2)}{=} \\
& (m \star l)^{\bullet} \in H^{\bullet} .
\end{aligned}
$$

${ }^{16}$ We shall refer to the $(i, j)$ cell of this product table by $\star_{(i, j)}$. 
$\star_{(1,2)}$ : By (6.15) the opposite of the statement is $x \star l \in H \cup H^{\bullet}$. Then, by claims (i) and (vi), $x=x \star t=x \star\left(l \star l^{\dagger}\right)=(x \star l) \star l^{\dagger} \in$ $\left(H \cup H^{\bullet}\right) \star H \subseteq H \cup H^{\bullet}$ follows using $\star_{(2,2)}$ and $\star_{(2,3)}$, a contradiction to $(6.15)$.

$\star_{(1,3)}$ : It follows from claim $(\mathrm{v})$ and $\star_{(1,2)}$.

$\star_{(1,1)}$ : Since $y \notin H, y=y \star f$ holds. Therefore, $x \star y=x \star(y \star f)=(x \star y) \star f$ follows, hence $x \star y$ cannot be in $H$.

We are ready to prove that $\mathbf{X}=(X, \dot{\wedge}, \dot{V}, \cdot, \rightarrow, t, t)$ is an odd involutive $\mathrm{FL}_{e^{-}}$ algebra. $(X, \dot{\wedge}, \dot{V})$ is a lattice. Indeed, all elements of $H^{\bullet}$ are meet-irreducible because of claim (iv), hence $X$ is closed under $\dot{\wedge}$ (clearly, $\dot{\wedge}$ is the restriction of $\wedge$ to $X$ ). Commutativity of $\dot{\vee}$ is straightforward, and using claim (iv) a moment's reflection shows that $\dot{V}$ is associative, too, and the absorption law holds for $\dot{\vee}$ and $\dot{\lambda}$. Commutativity of $\cdot$ is straightforward. $X$ is closed under - since by claim (iv), $(x \star y)_{\uparrow} \in H$ if $x \star y \in H^{\bullet} . t \in X$ holds by claim (iii). Since $t \in H, \star_{(2,1)}$ and $\star_{(2,2)}$ show that for $y \in X, t \star y \notin H^{\bullet}$ holds, hence $t y \stackrel{(6.3)}{=} h_{\mathbf{Y}}(t \star y) \stackrel{(6.2)}{=} t \star y$ and thus $t$ is the unit element for - over $X$ since it is the unit element for $\star$ over $Y$.

As for the associativity of $\cdot$, notice that

$$
\text { for } x, y \in Y, h_{\mathbf{Y}}(x \star y)=h_{\mathbf{Y}}(x) h_{\mathbf{Y}}(y) \text { holds. }
$$

Indeed, by (6.3), $h_{\mathbf{Y}}(x) h_{\mathbf{Y}}(y)=h_{\mathbf{Y}}\left(h_{\mathbf{Y}}(x) \star h_{\mathbf{Y}}(y)\right)$, and Table 3 readily confirms that $h_{\mathbf{Y}}\left(h_{\mathbf{Y}}(x) \star h_{\mathbf{Y}}(y)\right)=h_{\mathbf{Y}}(x \star y)$. Hence, $(X, \cdot)$ being the homomorphic image of a semigroup, is a semigroup.

Next we prove that $\cdot$ is residuated. For $x, y \in X, x \rightarrow y=\max \{z \in X:$ $x z \leq y\}$. Here

$$
x z \stackrel{(6.3)}{=}\left\{\begin{array}{lll}
x \star z & \text { if } & x \star z \notin H^{\bullet} \\
(x \star z)_{\uparrow} & \text { if } & x \star z \in H^{\bullet}
\end{array} .\right.
$$

If $x \star z \in H^{\bullet}$ then since $y \in X,(x \star z)_{\uparrow} \leq y$ holds if and only if $x \star z \leq y$ holds by claim (iv). Therefore, $\max \{z \in X: x z \leq y\}=\max \{z \in X: x \star z \leq y\}$ holds yielding $x \rightarrow y=x \rightarrow_{\star} y$. Since $x \rightarrow y \in X$, it also follows that $x \rightarrow y \stackrel{(6.2)}{=} h_{\mathbf{Y}}(x \rightarrow y)=h_{\mathbf{Y}}\left(x \rightarrow_{\star} y\right)$, as stated.

Involutivity of ${ }^{\dagger}$ is seen as follows. We will verify that

$$
x^{\dagger}=x_{\uparrow}^{\dagger} \text { if } x \in H, \text { and } x^{\dagger}=x^{\dagger} \text { if } x \in X \backslash H .
$$

This, combined with (3.1) and that $x^{\dagger} \in H$ if and only if $x \in H$ (shown by claims (vi) and (i) ) concludes the proof of the statement. Clearly, $x^{\dagger}=$ 
$x \rightarrow_{\star} t \geq x \rightarrow_{\star} f=x^{\star}$. Let $x \in H$. Equality cannot hold since $x \star x^{\dagger}=t$ by claim (i), whereas $x \star x^{t^{*}} \leq f<t$ by residuation and since $\mathbf{Y}$ is even. Assume that there exits $a \in X$ such that $x^{\dagger}>a>x^{\prime}$. Then $x^{\dagger}=x \rightarrow_{\star}$ $t \stackrel{L 3.1(i i)}{=}\left(x \star t^{t^{\star}}\right)^{\dagger} \stackrel{L 3.1(i)}{=}(x \star f)^{t^{*}}>a>x^{t^{\prime}}$, and hence $x \star f<a^{\dagger}<x$, a contradiction to claim (iv). Let $x \in X \backslash H$. If $x^{\dagger}>x^{\dagger}$ then, as above, $x \star f<x$ follows, a contradiction to $x \notin H$.

Finally, $\mathbf{X}$ is clearly odd, since the constant which defines the involution ${ }^{\dagger}$ is the unit element.

Next we prove that $\mathbf{H}$ is a cancellative subalgebra of $\mathbf{X}$. Indeed, $H$ is closed under $\star$, shown by $\star_{(2,2)}, t \in H$ holds by claim (iii), and $\mathbf{H}$ has an inverse operation $^{\dagger}$, see claims (i) and (vi), hence $\mathbf{H}$ is cancellative by claim (xi) in Lemma 3.1. It is a subalgebra of $\mathbf{X}$ by claim (iii).

Finally, we verify that $\mathbf{Y}$ is the $\mathbf{H}$-split of $\mathbf{X}$. Indeed, the universe is as expected, see (6.15). The elements of $H^{\bullet}$ are just below the respective elements of $H$, as they should be, see claim (iv). Finally we verify that $\star$ coincides with the product operation of the $\mathbf{H}$-split of $\mathbf{X}$.

If $x, y \in H$ then $x \star y \in H$ by $\star_{(2,2)}$. Hence $x \star y \notin H^{\bullet}$ by claim (ii), yielding $x \star y \stackrel{(6.3)}{=} x y \stackrel{(6.2)}{=} h_{\mathbf{Y}}(x) h_{\mathbf{Y}}(y)$, as required in (6.8).

If $h_{\mathbf{Y}}(x) h_{\mathbf{Y}}(y) \notin H$ then since $h_{\mathbf{Y}}(x), h_{\mathbf{Y}}(y) \in X$ and $X$ is closed under $\cdot, h_{\mathbf{Y}}(x) h_{\mathbf{Y}}(y) \in X \backslash H$ follows. Therefore, by $\star_{(1-1,2-2)}{ }^{17}$, either $h_{\mathbf{Y}}(x)$ or $h_{\mathbf{Y}}(y)$ (say $h_{\mathbf{Y}}(x)$ ) must be in $X \backslash H$, yielding $h_{\mathbf{Y}}(x) \stackrel{(6.2)}{=} x \in X \backslash H$. By the first row of Table 3 , for any $y \in Y, x \star y=x \star h_{\mathbf{Y}}(y)$. Since $H \not \supset h_{\mathbf{Y}}(x) h_{\mathbf{Y}}(y) \stackrel{(6.16)}{=} h_{\mathbf{Y}}(x \star y)$, it follows that $x \star y \notin H^{\bullet}$ and hence $x \star h_{\mathbf{Y}}(y) \notin H^{\bullet}$. Therefore, $x \star y=x \star h_{\mathbf{Y}}(y) \stackrel{(6.2)}{=} h_{\mathbf{Y}}\left(x \star h_{\mathbf{Y}}(y)\right) \stackrel{(6.3)}{=}$ $x h_{\mathbf{Y}}(y) \stackrel{(6.2)}{=} h_{\mathbf{Y}}(x) h_{\mathbf{Y}}(y)$, as required in (6.8).

Assume $\neg(x, y \in H)$ and $h_{\mathbf{Y}}(x) h_{\mathbf{Y}}(y) \in H$. By $(6.16), h_{\mathbf{Y}}(x \star y) \in H$, hence $x \star y \in H \cup H^{\bullet}$. Since $\neg(x, y \in H)$, by Table 3 it follows that $x \star y \in H^{\bullet}$. Table 3 and $x \star y \in H^{\bullet}$ also implies that either $x, y \in X \backslash H$, or at least one of $x$ and $y$ is in $H^{\bullet}$ and the other is in $H \cup H^{\bullet}$. In all these cases, by Table 3 and (6.2) it follows that $x \star y=h_{\mathbf{Y}}(x) \star h_{\mathbf{Y}}(y)$. Hence $h_{\mathbf{Y}}(x) \star h_{\mathbf{Y}}(y) \in H^{\bullet}$ and it yields $\left(h_{\mathbf{Y}}(x) \star h_{\mathbf{Y}}(y)\right)_{\uparrow} \stackrel{(6.2)}{=} h_{\mathbf{Y}}\left(h_{\mathbf{Y}}(x) \star\right.$ $\left.h_{\mathbf{Y}}(y)\right) \stackrel{(6.3)}{=} h_{\mathbf{Y}}(x) h_{\mathbf{Y}}(y)$, that is, $x \star y=h_{\mathbf{Y}}(x) \star h_{\mathbf{Y}}(y)=\left(h_{\mathbf{Y}}(x) h_{\mathbf{Y}}(y)\right)_{\downarrow}$, as required in (6.8).

${ }^{17}$ This notation refers to the submatrix containing $\star_{(1,1)}, \star_{(1,2)}, \star_{(2,1)}, \star_{(2,2)}$. 
(b): Since $h_{\mathbf{Y}}$ maps onto $\mathbf{X}$, to prove that $\mathbf{X}$ is cancellative it suffices to prove that each element of $h_{\mathbf{Y}}(\mathbf{Y})$ has inverse, which holds since for $x \in Y$, $h_{\mathbf{Y}}(x) h_{\mathbf{Y}}\left(x^{t^{\star}}\right) \stackrel{(6.16)}{=} h_{\mathbf{Y}}\left(x \star x^{t^{\star}}\right)=h_{\mathbf{Y}}(f) \stackrel{\mathbf{Y}}{=} \stackrel{\text { is even }}{=} h_{\mathbf{Y}}\left(t_{\downarrow}\right) \stackrel{t \in H,(6.7)}{=} t$.

(c): It is obvious that $h_{\mathbf{Y}}$ preserves the unit element and the falsum constant. The definitions in (6.2)-(6.4) readily yield that $h_{\mathbf{Y}}$ preserves the meet, the join, the product and the residual operation.

\section{Bunches of Layer Algebras vs. Bunches of Layer Groups}

We introduce the notion of bunches of layer groups, and show that every bunch of layer algebras can be represented by a unique bunch of layer groups.

DeFinition 7.1. Let $\left(\kappa, \leq_{\kappa}\right)$ be a totally ordered set with least element $t$, and let an ordered triple $\left\langle\bar{\kappa}_{I}, \bar{\kappa}_{J},\{t\}\right\rangle$ be a partition of $\kappa$, where $\bar{\kappa}_{I}$ and $\bar{\kappa}_{J}$ can also be empty. Define $\kappa_{O}, \kappa_{J}$, and $\kappa_{I}$ by one of the rows of Table 1 , and let $\boldsymbol{\kappa}=\left\langle\kappa_{O}, \kappa_{J}, \kappa_{I}, \leq_{\kappa}\right\rangle$. Let $\boldsymbol{G}_{u}=\left(G_{u}, \preceq_{u}, \cdot_{u},{ }^{-1_{u}}, u\right)$ be a family of abelian $o$-groups indexed by elements of $\kappa$, an let $\boldsymbol{H}_{u}=\left(H_{u}, \preceq_{u}, \cdot{ }_{u},{ }^{-1_{u}}, u\right)$ be a family of abelian $o$-groups indexed by elements of $\kappa_{I}$, such that

$$
\begin{gathered}
\text { for } u \in \kappa_{J}, \boldsymbol{G}_{u} \text { is discrete, } \\
\text { for } u \in \kappa_{I}, \boldsymbol{H}_{u} \leq \boldsymbol{G}_{u},
\end{gathered}
$$

and such that for $u, v \in \kappa, u \leq_{\kappa} v$, there exist

$$
\text { homomorphisms } \varsigma_{u \rightarrow v}: G_{u} \rightarrow G_{v}
$$

satisfying

(G1) $\varsigma_{u \rightarrow u}=i d_{G_{u}}$ and $\varsigma_{v \rightarrow w} \circ \varsigma_{u \rightarrow v}=\varsigma_{u \rightarrow w} \quad$ (direct system property),

(G2) for $u \in \kappa_{J}, \varsigma_{u \rightarrow v}(u)=\varsigma_{u \rightarrow v}\left(u_{\downarrow_{u}}\right)$,

(G3) for $v \in \kappa_{I}, \varsigma_{u \rightarrow v}$ maps into $H_{v}$.

Call $\mathcal{G}=\left\langle\boldsymbol{G}_{u}, \boldsymbol{H}_{u}, \varsigma_{u \rightarrow v}\right\rangle_{\kappa}$ a bunch of layer groups.

LEMMA 7.2. The following statements hold true.

(1) Given a bunch of layer algebras $\mathcal{A}=\left\langle\mathbf{X}_{u}, \rho_{u \rightarrow v}\right\rangle_{\kappa}$ with $\boldsymbol{\kappa}=$ $\left\langle\kappa_{O}, \kappa_{J}, \kappa_{I}, \leq_{\kappa}\right\rangle$,

$$
\mathcal{G}_{\mathcal{A}}=\left\langle\boldsymbol{G}_{u}, \boldsymbol{H}_{u}, \varsigma_{u \rightarrow v}\right\rangle_{\kappa}
$$


is bunch of layer groups, where

$$
\boldsymbol{G}_{u}=\left(G_{u}, \preceq_{u}, \cdot_{u},{ }^{-1_{u}}, u\right)= \begin{cases}\lambda\left(\mathbf{X}_{u}\right) & \text { if } u \in \kappa_{o} \\ \lambda\left(\mathbf{X}_{u \boldsymbol{\uparrow}}\right) & \text { if } u \in \kappa_{J}, \\ \lambda\left(\pi_{1}\left(\mathbf{X}_{u}\right)\right) & \text { if } u \in \kappa_{I}\end{cases}
$$

for $u \in \kappa_{I}$,

$$
\boldsymbol{H}_{u}=\left(H_{u}, \preceq_{u},{ }^{\cdot},{ }^{-1_{u}}, u\right)=\lambda\left(\pi_{2}\left(\mathbf{X}_{u}\right)\right),
$$

$\kappa=\kappa_{o} \cup \kappa_{J} \cup \kappa_{I}$, and for $u, v \in \kappa$ such that $u \leq_{\kappa} v, \varsigma_{u \rightarrow v}: G_{u} \rightarrow G_{v}$ is defined by

$$
\varsigma_{u \rightarrow v}=\left.\rho_{u \rightarrow v}\right|_{G_{u}} .
$$

Call $\mathcal{G}_{\mathcal{A}}$ the bunch of layer groups derived from $\mathcal{A}$.

(2) Given a bunch of layer groups $\mathcal{G}=\left\langle\boldsymbol{G}_{u}, \boldsymbol{H}_{u}, \varsigma_{u \rightarrow v}\right\rangle_{\boldsymbol{\kappa}}$ with $\boldsymbol{\kappa}=$ $\left\langle\kappa_{o}, \kappa_{J}, \kappa_{I}, \leq_{\kappa}\right\rangle$,

$$
\mathcal{A}_{\mathcal{G}}=\left\langle\mathbf{X}_{u}, \rho_{u \rightarrow v}\right\rangle_{\kappa}
$$

is bunch of layer algebras, called the bunch of layer algebras derived from $\mathcal{G}$, where

$\mathbf{X}_{u}=\left(X_{u}, \leq_{u}, \cdot_{u}, \rightarrow_{u}, u, u^{\prime}{ }^{u}\right)=\left\{\begin{array}{ll}\iota\left(\boldsymbol{G}_{u}\right) & \text { if } u \in \kappa_{o} \\ \iota\left(\boldsymbol{G}_{u}\right)_{\downarrow} & \text { if } u \in \kappa_{J} \\ S p\left(\iota\left(\boldsymbol{G}_{u}\right), \iota\left(\boldsymbol{H}_{u}\right)\right), & \text { if } u \in \kappa_{I}\end{array}\right.$,

$\kappa=\kappa_{o} \cup \kappa_{J} \cup \kappa_{I}$, and for $u, v \in \kappa$ such that $u \leq_{\kappa} v, \rho_{u \rightarrow v}: X_{u} \rightarrow X_{v}$ is defined by

$$
\rho_{u \rightarrow v}= \begin{cases}\varsigma_{u \rightarrow v} & \text { if } u \notin \kappa_{I} \\ \varsigma_{u \rightarrow v} & \circ h_{u} \text { if } v>u \in \kappa_{I}, \\ i d_{X_{u}} & \text { if } v=u \in \kappa_{I}\end{cases}
$$

where $h_{u}$ is the canonical homomorphism of $\mathbf{X}_{u}$.

(3) Given a bunch of layer groups $\mathcal{G}$, it holds true that $\mathcal{G}_{\left(\mathcal{A}_{\mathcal{G}}\right)}=\mathcal{G}$, and given a bunch of layer algebras $\mathcal{A}$, it holds true that $\mathcal{A}_{\left(\mathcal{G}_{\mathcal{A}}\right)}=\mathcal{A}$.

Proof. (1): For $u \in \kappa, \boldsymbol{G}_{u}$ defined by (7.2) is an abelian o-group. Indeed, being totally ordered is granted since so is the original algebra. If $u \in \kappa_{o}$ then see (4.1) and Lemma 3.2, if $u \in \kappa_{J}$ then see (4.1) and Lemmas 5.2 and 3.2 , it also confirms (7.1), whereas if $u \in \kappa_{I}$ then see (4.1), Theorem 6.3 and Lemma 3.2. The $\varsigma$ 's defined in (7.4) satisfy 
(G1) for $x \in G_{u}, \varsigma_{u \rightarrow u}(x) \stackrel{(7.4)}{=} \rho_{u \rightarrow u}(x) \stackrel{(A 1)}{=} x$ and $\left(\varsigma_{v \rightarrow w} \circ \varsigma_{u \rightarrow v}\right)(x) \stackrel{(7.4)}{=}$ $\left(\rho_{v \rightarrow w} \circ \rho_{u \rightarrow v}\right)(x) \stackrel{(\mathrm{A} 1)}{=} \rho_{u \rightarrow w}(x) \stackrel{(7.4)}{=} \varsigma_{u \rightarrow w}(x)$.

(G2) Let $u \in \kappa_{J}$. Then $\varsigma_{u \rightarrow v}\left(u_{\downarrow_{u}}\right) \stackrel{(7.4)}{=} \rho_{u \rightarrow v}\left(u_{\downarrow_{u}}\right) \stackrel{(4.1)}{=} \rho_{u \rightarrow v}\left(u^{{ }^{u}}\right) \stackrel{(\mathrm{A} 2)}{=}$ $\rho_{u \rightarrow v}(u) \stackrel{(7.4)}{=} \varsigma_{u \rightarrow v}(u)$.

(G3) Let $v \in \kappa_{I}$. Then $\mathbf{X}_{v}=S p\left(\pi_{1}\left(\mathbf{X}_{v}\right), \pi_{2}\left(\mathbf{X}_{v}\right)\right)$ holds by Theorem 6.3. For $x \in G_{u}, \varsigma_{u \rightarrow v}(x) \stackrel{(7.4)}{=} \rho_{u \rightarrow v}(x) \in X_{v}$, and by claim $(\mathrm{C} 1)$ in the proof of claim (2) of Lemma 4.2, $\rho_{u \rightarrow v}(x)$ is invertible in $\mathbf{X}_{v}$. Therefore, $\rho_{u \rightarrow v}(x)$ is an element of $\pi_{2}\left(\mathbf{X}_{v}\right) \stackrel{(7.3)}{=} \mathbf{H}_{u}$ by claim (c) in Theorem 6.3 .

(2): $\mathbf{X}_{u}$ defined in (7.5) is an involutive $\mathrm{FL}_{e}$-chain satisfying (4.1): if $u \in \kappa_{o}$ then see Lemma 3.2, if $u \in \kappa_{J}$ then see Lemmas 3.2 and 5.2, if $u \in \kappa_{I}$ then see Lemma 3.2 and Theorem 6.3.

The $\rho$ 's defined in (7.6) are well defined since $X_{u}=G_{u}$ holds by (7.5) if $u \notin \kappa_{I}$, and if $u \in \kappa_{I}$ then $h_{u}$ maps to the universe of $\iota\left(\boldsymbol{G}_{u}\right)$ (see (6.2)), which is $G_{u}$.

The $\rho$ 's are residuated lattice homomorphisms, since the (totally ordered group) homomorphisms $\varsigma_{u \rightarrow v}$ from $\boldsymbol{G}_{u}$ to $\boldsymbol{G}_{v}$ naturally extend to homomorphisms from the residuated lattice reduct of $\iota\left(\boldsymbol{G}_{u}\right)$ to the residuated lattice reduct of $\iota\left(\boldsymbol{G}_{v}\right)$ via claim (2) of Lemma 3.3, and hence $\rho_{u \rightarrow v}$ can be regarded as the composition of residuated lattice homomorphisms ( $\varsigma$ 's and $h$ 's).

The $\rho$ 's satisfy

(A1): Notice that for $u<v$,

$$
\rho_{u \rightarrow v} \operatorname{maps} X_{u} \text { to } G_{v},
$$

since so does $\varsigma_{u \rightarrow v}$. Over $X_{u}$,

$$
\rho_{u \rightarrow u} \stackrel{(7.6)}{=}\left\{\begin{array}{ll}
\varsigma_{u \rightarrow u} \stackrel{(G 1)}{=} i d_{G_{u}} \stackrel{(7.5)}{=} i d_{X_{u}} & \text { if } \quad u \notin \kappa_{I} \\
i d_{X_{u}} & \text { if } v=u \in \kappa_{I}
\end{array} .\right.
$$

Therefore, it suffices to prove the other condition in (A1) for $u<v<w$ only:

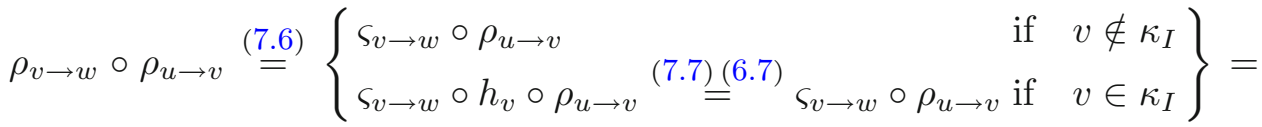

$$
\begin{aligned}
& \varsigma_{v \rightarrow w} \circ \rho_{u \rightarrow v} \stackrel{(7.6)}{=}\left\{\begin{array}{lll}
\varsigma_{v \rightarrow w} \circ \varsigma_{u \rightarrow v} \stackrel{(\mathrm{G} 1)}{=} \varsigma_{u \rightarrow w} & \text { if } & u \notin \kappa_{I} \\
\varsigma_{v \rightarrow w} \circ \varsigma_{u \rightarrow v} \circ h_{u} \stackrel{(\mathrm{G} 1)}{=} \varsigma_{u \rightarrow w} \circ h_{u} \text { if } & u \in \kappa_{I}
\end{array}\right\} \stackrel{(7.6)}{=} \rho_{u \rightarrow w} .
\end{aligned}
$$


(A2): For $u \notin \kappa_{o}$ we have already seen that

$$
\mathbf{X}_{u} \text { is even, }
$$

therefore, $\rho_{u \rightarrow v}\left(u^{\mu}\right) \stackrel{(7.6)}{=}$

$$
\begin{cases}\varsigma_{u \rightarrow v}\left(u^{u^{u}}\right) \stackrel{(7.8)}{=} \varsigma_{u \rightarrow v}\left(u_{\downarrow u}\right) \stackrel{(\mathrm{G} 2)}{=} \varsigma_{u \rightarrow v}(u) \stackrel{(7.6)}{=} \rho_{u \rightarrow v}(u) \text { if } & u \in \kappa_{J}, \\ \left(\varsigma_{u \rightarrow v} \circ h_{u}\right)\left(u^{\prime}\right) \stackrel{(6.7)}{=} \varsigma_{u \rightarrow v}\left(u_{\uparrow}^{\prime}\right) \stackrel{(7.8)}{=} \varsigma_{u \rightarrow v}(u) & \text { if } u \in \kappa_{I} . \\ \stackrel{(6.7)}{=}\left(\varsigma_{u \rightarrow v} \circ h_{u}\right)(u) \stackrel{(7.6)}{=} \rho_{u \rightarrow v}(u) & \end{cases}
$$

(3): If $u=\kappa_{o}$ then $\lambda\left(\iota\left(\boldsymbol{G}_{u}\right)\right)=\boldsymbol{G}_{u}$ and $\iota\left(\lambda\left(\mathbf{X}_{u}\right)\right)=\mathbf{X}_{u}$ by Lemma 3.2. If $u=\kappa_{J}$ then $\lambda\left(\iota\left(\boldsymbol{G}_{u}\right)_{\downarrow \uparrow}\right)=\lambda\left(\iota\left(\boldsymbol{G}_{u}\right)\right)=\boldsymbol{G}_{u}$ and $\iota\left(\lambda\left(\mathbf{X}_{u \boldsymbol{\uparrow}}\right)\right)_{\downarrow}=\mathbf{X}_{u \boldsymbol{\uparrow}}=$ $\mathbf{X}_{u}$ follow from Lemma 5.2 and Lemma 3.2. In these two cases $G_{u}=X_{u}$, thus it is obvious from $(7.6)$ and (7.4) that $\mathcal{G}_{\left(\mathcal{A}_{\mathcal{G}}\right)}$ and $\mathcal{G}$ have the same homomorphisms (the same $\varsigma$ 's) from the $u^{\text {th }}$-layer, and that $\mathcal{A}_{\left(\mathcal{G}_{\mathcal{A}}\right)}$ and $\mathcal{A}$ have the same homomorphisms (the same $\rho$ 's) from the $u^{\text {th }}$-layer. If $u \in \kappa_{I}$ then $\lambda\left(\pi_{1}\left(S p\left(\iota\left(\boldsymbol{G}_{u}\right), \iota\left(\boldsymbol{H}_{u}\right)\right)\right)\right)=\lambda\left(\iota\left(\boldsymbol{G}_{u}\right)\right)=\boldsymbol{G}_{u}, \lambda\left(\pi_{2}\left(S p\left(\iota\left(\boldsymbol{G}_{u}\right), \iota\left(\boldsymbol{H}_{u}\right)\right)\right)\right)=$ $\lambda\left(\iota\left(\boldsymbol{H}_{u}\right)\right)=\boldsymbol{H}_{u}$ and $\operatorname{Sp}\left(\iota\left(\lambda\left(\pi_{1}\left(\mathbf{X}_{u}\right)\right)\right), \iota\left(\lambda\left(\pi_{2}\left(\mathbf{X}_{u}\right)\right)\right)\right)=S p\left(\pi_{1}\left(\mathbf{X}_{u}\right), \pi_{2}\left(\mathbf{X}_{u}\right)\right)$ $=\mathbf{X}_{u}$ follow from Theorem 6.3 and Lemma 3.2. As for the homomorphisms, $h_{u}$ maps $X_{u}$ to $G_{u}$ by (7.2), hence the composition $\varsigma_{u \rightarrow v} \circ h_{u}$ is well-defined. By the construction in Definition 6.1, $G_{u}$ can also be regarded as a subset of $X_{u}$, and $h_{u}$ is the identity mapping on $G_{u}$ by (6.2). Therefore, $\left.\left(\varsigma_{u \rightarrow v} \circ h_{u}\right)\right|_{G_{u}}=\varsigma_{u \rightarrow v}$ holds on the one hand. On the other hand, to prove $\left.\rho_{u \rightarrow v}\right|_{G_{u}} \circ h_{u}=\rho_{u \rightarrow v}$, first notice that for $x \in X_{u} \backslash G_{u}$,

$$
\rho_{u \rightarrow v}\left(x_{\uparrow_{u}}\right)=\rho_{u \rightarrow v}(x) .
$$

Indeed, $\rho_{u \rightarrow v}\left(x_{\uparrow_{u}}\right)=\rho_{u \rightarrow v}\left(x_{\uparrow_{u}} \cdot{ }_{u} u\right) \stackrel{(4.2)}{=} \rho_{u \rightarrow v}\left(x_{\uparrow_{u}}\right) \cdot_{v} \rho_{u \rightarrow v}(u) \stackrel{(A 2)}{=}$ $\rho_{u \rightarrow v}\left(x_{\uparrow_{u}}\right) \cdot v \rho_{u \rightarrow v}\left(u^{{ }^{\mu}}\right) \quad X_{u}$ is even, see (4.1) $\quad \rho_{u \rightarrow v}\left(x_{\uparrow_{u}}\right) \cdot v \rho_{u \rightarrow v}\left(u_{\downarrow_{u}}\right) \stackrel{(4.2)}{=}$ $\rho_{u \rightarrow v}\left(x_{\uparrow_{u}} \cdot{ }_{u} u_{\downarrow_{u}}\right) \stackrel{(6.8)}{=} \rho_{u \rightarrow v}\left(x_{\uparrow_{u} \downarrow_{u}}\right)=\rho_{u \rightarrow v}(x)$. Therefore, $\left(\left.\rho_{u \rightarrow v}\right|_{G_{u}} \circ h_{u}\right)(x)$ $\stackrel{(6.7)}{=}$

$\stackrel{(6.7)}{=}\left\{\begin{array}{lll}\left(\left.\rho_{u \rightarrow v}\right|_{G_{u}}\right)(x)=\rho_{u \rightarrow v}(x) & \text { if } & x \in G_{u} \\ \left(\left.\rho_{u \rightarrow v}\right|_{G_{u}}\right)\left(x_{\uparrow_{u}}\right) \stackrel{x_{\uparrow u} \in H_{u} \subseteq G_{u}}{=} \rho_{u \rightarrow v}\left(x_{\uparrow u}\right) \stackrel{(7.9)}{=} \rho_{u \rightarrow v}(x) \text { if } & x \in X_{u} \backslash G_{u}\end{array}\right.$.

\section{The Representation Theorem}

The main theorem of the paper is a representation theorem of odd or even involutive $\mathrm{FL}_{e}$-chains by bunches of layer groups. Lemmas 4.2 and 7.2 prove 
Theorem 8.1. The second part of Theorem 8.1 presents the direct constructional correspondence between odd or even involutive $\mathrm{FL}_{e}$-chains and bunches of layer-groups, that is, one without referring to the intermediate explanatory step of layer algebras.

THEOREM 8.1. For every odd or even involutive $F L_{e}$-chain $\mathbf{X}$ there exists a unique bunch of layer groups $\mathcal{G}$ such that $\mathbf{X}$ is the involutive $F L_{e}$-chain derived from the bunch of layer algebras derived from $\mathcal{G}$, in notation, $\mathbf{X}=$ $\mathcal{X}_{\mathcal{A}_{\mathcal{G}}}$. Conversely, for every bunch of layer groups $\mathcal{G}$ there exists a unique odd or even involutive $F L_{e}$-chain $\mathbf{X}$ such that $\mathcal{G}$ is the bunch of layer groups derived from the bunch of layer algebras of $\mathbf{X}$, in notation, $\mathcal{G}=\mathcal{G}_{\mathcal{A}_{\mathbf{X}}}$. In more details:

(A) Given an odd or an even involutive $F L_{e}$-chain $\mathbf{X}=(X, \leq, \cdot, \rightarrow, t, f)$ with residual complement operation ',

$$
\mathcal{G}_{\mathbf{X}}=\left\langle\boldsymbol{G}_{u}, \boldsymbol{H}_{u}, \varsigma_{u \rightarrow v}\right\rangle_{\left\langle\kappa_{o}, \kappa_{J}, \kappa_{I}, \leq_{\kappa}\right\rangle}
$$

is bunch of layer groups, called the bunch of layer groups of $\mathbf{X}$, where $\kappa=\{x \rightarrow x: x \in X\}=\{u \geq t: u$ is idempotent $\}$ is ordered by $\leq$, $\bar{\kappa}_{I}=\left\{u \in \kappa \backslash\{t\}: u^{\prime}\right.$ is idempotent $\}$, $\bar{\kappa}_{J}=\left\{u \in \kappa \backslash\{t\}: u^{\prime}\right.$ is not idempotent $\}$, $\kappa_{0}, \kappa_{J}, \kappa_{I}$ are defined by Table 4 ,

$$
\begin{array}{ll}
\boldsymbol{G}_{u}=\left(G_{u}, \leq, \cdot,-1, u\right) & \text { if } u \notin \kappa_{I}, \\
\boldsymbol{G}_{u}=\left(G_{u}, \leq, \cdot u,-1, u\right) & \text { if } u \in \kappa_{I}, \\
\boldsymbol{H}_{u}=\left(H_{u}, \leq, \cdot,^{-1}, u\right) & \text { if } u \in \kappa_{I},
\end{array}
$$

where $X_{u}=\{x \in X: x \rightarrow x=u\}, H_{u}=\left\{x \in X_{u}: x u^{\prime}<x\right\}=\{x \in$ $X_{u}: x$, is u-invertible in $\left.X_{u}^{18}\right\}, \dot{H}_{u}=\left\{x u^{\prime}: x \in H_{u}\right\}$,

$$
\begin{aligned}
& G_{u}= \begin{cases}X_{u} & \text { if } u \notin \kappa_{I} \\
X_{u} \backslash H_{u}^{\bullet} & \text { if } u \in \kappa_{I}\end{cases} \\
& x \cdot u y=(x y \rightarrow u) \rightarrow u \\
& x^{-1}=x \rightarrow u,
\end{aligned}
$$

(B) Given a bunch of layer groups $\mathcal{G}=\left\langle G_{u}, H_{u}, \varsigma_{u \rightarrow v}\right\rangle_{\left\langle\kappa_{o}, \kappa_{J}, \kappa_{I}, \leq_{\kappa}\right\rangle}$ with $\boldsymbol{G}_{u}=\left(G_{u}, \preceq_{u},{ }_{u},{ }^{-1_{u}}, u\right)$

$$
\mathbf{X}_{\mathcal{G}}=\left(X, \leq, \cdot, \rightarrow, t, t^{\prime}\right)
$$

\footnotetext{
${ }^{18}$ There exists $y \in X_{u}$ such that $x y=u$.
} 
is an involutive $F L_{e}$-chain with residual complement', called the involutive $F L_{e}$-chain of $\mathcal{X}$, where $\kappa=\kappa_{o} \cup \kappa_{J} \cup \kappa_{I}$, for $u \in \kappa$,

$$
X_{u}= \begin{cases}G_{u} & \text { if } u \notin \kappa_{I}, \\ G_{u} \cup H_{u}^{\bullet} & \text { if } u \in \kappa_{I},\end{cases}
$$

(where $H_{u}^{\bullet}=\left\{h^{\bullet}: h \in H_{u}\right\}$ is a copy of $H_{u}$ which is disjoint from $\left.G_{u}\right)$,

$$
X=\bigcup_{u \in \kappa} X_{u}
$$

if $u \notin \kappa_{I}$ then $\leq_{u}=\preceq_{u}$, if $u \in \kappa_{I}$ then $\leq_{u}$ extends $\preceq_{u}$ to $X_{u}$ by letting

$a^{\bullet}<_{u} b^{\bullet}$ and $x<_{u} a^{\bullet}<_{u} y$ if $a, b \in H_{u}, x, y \in G_{u}, a \prec_{u} b, x \prec_{u} a \preceq_{u} y$, for $v \in \kappa, \rho_{v}: X \rightarrow X$ is defined by

$$
\begin{aligned}
& \rho_{v}(x)= \begin{cases}\varsigma_{u \rightarrow v}(x) & \text { if } x \in G_{u} \text { and } u<_{\kappa} v, \\
x & \text { if } x \in G_{u} \text { and } u \geq_{\kappa} v,\end{cases} \\
& \rho_{v}(\dot{x})= \begin{cases}\varsigma_{u \rightarrow v}(x) & \text { if } x \bullet H_{u}^{\bullet} \text { and } \kappa_{I} \ni u<_{\kappa} v, \\
x^{\bullet} & \text { if } x \in H_{u}^{\bullet} \text { and } \kappa_{I} \ni u \geq_{\kappa} v,\end{cases}
\end{aligned}
$$

by denoting for $u, v \in \kappa, u v=\max _{\kappa}(u, v)$, for $x \in X_{u}$ and $y \in X_{v}$,

$$
x<y \text { iff } \rho_{u v}(x)<_{u v} \rho_{u v}(y) \text { or } \rho_{u v}(x)=\rho_{u v}(y) \text { and } u<_{\kappa} v,
$$

for $u \in \kappa_{I}, h_{u}: X_{u} \rightarrow G_{u}$,

$$
\begin{array}{ll}
h_{u}(x)=x & \text { if } \quad x \in G_{u}, \\
h_{u}\left(x^{\bullet}\right)=x & \text { if } \quad x^{\bullet} \in H_{u}^{\bullet},
\end{array}
$$

for $x, y \in X_{u}$,

$x \bullet \bullet_{u} y=\left\{\begin{array}{ll}\left(h_{u}(x) \cdot{ }_{u} h_{u}(y)\right)^{\bullet} & \text { if } u \in \kappa_{I}, h_{u}(x) \cdot{ }_{u} h_{u}(y) \in H_{u} \text { and } \neg\left(x, y \in H_{u}\right) \\ h_{u}(x) \cdot{ }_{u} h_{u}(y) & \text { if } u \in \kappa_{I}, h_{u}(x) \cdot{ }_{u} h_{u}(y) \notin H_{u} \text { or } x, y \in H_{u} \\ x \cdot{ }_{u} y & \text { if } u \notin \kappa_{I}\end{array}\right.$, for $x \in X_{u}$ and $y \in X_{v}$,

$$
x y=\rho_{u v}(x) \bullet{ }_{u v} \rho_{u v}(y),
$$

for $x \in X$,

$$
\begin{aligned}
& \left(x^{\bullet}\right)^{\prime}=\left\{x^{-1} \text { if } u \in \kappa_{I} \text { and } x^{\bullet} \in H_{u}^{\bullet}\right. \\
& x^{\prime}=\left\{\begin{array}{ll}
\left(x^{-1}\right)^{\bullet} & \text { if } u \in \kappa_{I} \text { and } x \in H_{u} \\
x^{-1} & \text { if } u \in \kappa_{I} \text { and } x \in G_{u} \backslash H_{u} \\
x^{-1} & \text { if } u \in \kappa_{o} \text { and } x \in G_{u} \\
x^{-1} \downarrow & \text { if } u \in \kappa_{J} \text { and } x \in G_{u}
\end{array},\right.
\end{aligned}
$$


Table 4. Three cases - three classes

\begin{tabular}{llll}
\hline$\kappa_{o}$ & $\kappa_{J}$ & $\kappa_{I}$ & \\
\hline$\{\mathrm{t}\}$ & $\bar{\kappa}_{J}$ & $\bar{\kappa}_{I}$ & if $\mathbf{X}$ is odd \\
$\emptyset$ & $\bar{\kappa}_{J} \cup\{t\}$ & $\bar{\kappa}_{I}$ & if $\mathbf{X}$ is even and $f$ is not idempotent \\
$\emptyset$ & $\bar{\kappa}_{J}$ & $\bar{\kappa}_{I} \cup\{t\}$ & if $\mathbf{X}$ is even and $f$ is idempotent \\
\hline
\end{tabular}

for $x, y \in X$,

$$
x \rightarrow y=\left(x y^{\prime}\right)^{\prime},
$$

$\rightarrow$ is the residual operation of $\cdot$,

$t$ is the least element of $\kappa$,

$f$ is the residual complement of ,

and is given by

$$
t^{\prime}= \begin{cases}\left(t^{-1}\right)^{\bullet} & \text { if } u \in \kappa_{I} \\ t^{-1} & \text { if } u \in \kappa_{o} \\ t^{-1} \downarrow & \text { if } u \in \kappa_{J}\end{cases}
$$

In addition,

$$
\rho_{v}(x)=v x \quad \text { for } v \in \kappa \text { and } x \in X,
$$

$\mathbf{X}_{\mathcal{X}}$ is odd if $t \in \kappa_{o}$, even with a non-idempotent falsum if $t \in \kappa_{J}$, and even with an idempotent falsum if $t \in \kappa_{I}$.

(C) Items $(\mathbf{A})$ and $(\mathbf{B})$ describe a one-to-one correspondence between the class containing all odd and all even involutive $F L_{e}$-chains and the class of bunches of layer groups: given a bunch of layer groups $\mathcal{X}$ it holds true that $\mathcal{X}_{\left(\mathbf{X}_{\mathcal{X}}\right)}=\mathcal{X}$, and given an odd or even involutive $F L_{e}$-chain $\mathbf{X}$ it holds true that $\mathbf{X}_{\left(\mathcal{X}_{\mathbf{X}}\right)}=\mathbf{X}$.

EXAMPLE 8.2. We present the bunch representation of a few known structures, among which are the two extremal classes (abelian o-groups and odd Sugihara chains) mentioned in the introduction. Denote by $\mathbb{1}$ the trivial (one-element) group.

- If $\boldsymbol{G}$ is an abelian o-group then $\boldsymbol{G}=\mathbf{X}_{\mathcal{G}}$ where

$$
\mathcal{G}=\langle\boldsymbol{G}, \emptyset, \emptyset\rangle_{\left\langle\{t\}, \emptyset, \emptyset, \leq_{\kappa}\right\rangle} .
$$

- Even Sugihara chains are exactly the algebras $\mathbf{X}_{\mathcal{G}}$, where

$$
\mathcal{G}=\left\langle\mathbb{1}_{u}, \mathbb{1}_{u}, \varsigma_{u \rightarrow v}\right\rangle_{\left\langle\emptyset, \emptyset, \kappa, \leq_{\kappa}\right\rangle} .
$$


- Odd Sugihara chains are exactly the algebras $\mathbf{X}_{\mathcal{G}}$, where

$$
\mathcal{G}=\left\langle\mathbb{1}_{u}, \mathbb{1}_{u}, \varsigma_{u \rightarrow v}\right\rangle_{\left\langle\{t\}, \emptyset, \kappa \backslash\{t\}, \leq_{\kappa}\right\rangle} .
$$

- Finite partial sublex products of abelian o-groups have been shown in $[31,32]$ to be exactly those odd involutive $\mathrm{FL}_{e}$-chains which have finitely many positive idempotent elements. These are exactly the algebras $\mathbf{X}_{\mathcal{G}}$, where $\kappa$ is finite in

$$
\mathcal{G}=\left\langle\boldsymbol{G}_{u}, \boldsymbol{H}_{u}, \varsigma_{u \rightarrow v}\right\rangle_{\left\langle\{t\}, \bar{\kappa}_{J}, \bar{\kappa}_{I}, \leq_{\kappa}\right\rangle} \cdot
$$

- Algebras which can be constructed by the involutive ordinal sum construction of [28] are exactly the algebras $\mathbf{X}_{\mathcal{G}}$, where

$$
\mathcal{G}=\left\langle\boldsymbol{G}_{u}, \mathbb{1}_{u}, \varsigma_{u \rightarrow v}\right\rangle_{\left\langle\{t\}, \emptyset, \kappa \backslash\{t\}, \leq_{\kappa}\right\rangle} \cdot
$$

By (G3), also the homomorphisms are trivial.

- Algebras which can be constructed by the consecutive application of the ordinal sum construction as defined in [18] are exactly the algebras $\mathbf{X}_{\mathcal{G}}$, where $\kappa$ is finite in

$$
\mathcal{G}=\left\langle\boldsymbol{G}_{u}, \mathbb{1}_{u}, \varsigma_{0}^{u \rightarrow v}\right\rangle_{\left\langle\{t\}, \emptyset, \kappa \backslash\{t\}, \leq_{\kappa}\right\rangle} .
$$

By (G3), also the homomorphisms are trivial.

REMARK 8.3. If $\mathbf{X}$ is densely ordered in Theorem 8.1 then the $\boldsymbol{H}_{u}$ 's in the representation $\mathcal{G}=\left\langle\boldsymbol{G}_{u}, \boldsymbol{H}_{u}, \varsigma_{u \rightarrow v}\right\rangle_{\boldsymbol{\kappa}}$ of $\mathbf{X}$ are uniquely determined by the rest of $\mathcal{G}$. Therefore, if $\mathbf{X}$ is densely ordered then the representation of $\mathbf{X}$ by layer groups can be written in a simpler form of $\left\langle\boldsymbol{G}_{u}, \varsigma_{u \rightarrow v}\right\rangle_{\left\langle\kappa_{o}, \kappa_{J}, \kappa_{I}, \leq_{\kappa}\right\rangle}$. To prove it we state that for $u \in \kappa_{I}$,

$$
H_{u}=\bigcup_{\kappa \ni s<{ }_{\kappa} u} \varsigma_{s \rightarrow u}\left(G_{s}\right) .
$$

Indeed, $H_{u} \supseteq \bigcup_{\kappa \ni s<{ }_{\kappa} u} \varsigma_{s \rightarrow u}\left(G_{s}\right)$ follows from (G3). Since $u \in \kappa_{I}$, and since $\boldsymbol{\kappa}$ is the same in $\mathcal{G}_{\mathcal{A}_{\mathbf{X}}}$ and in $\mathcal{A}_{\mathbf{X}}$, the $u^{\text {th }}$-layer algebra $\mathbf{X}_{u}$ of $\mathbf{X}$ has idempotent falsum constant $u^{\prime}$ by (4.1). Therefore, by Theorem 6.3, $\mathbf{X}_{u}=S p\left(\pi_{1}\left(\mathbf{X}_{u}\right), \pi_{2}\left(\mathbf{X}_{u}\right)\right)$, where $\pi_{2}\left(\mathbf{X}_{u}\right)=\boldsymbol{H}_{u}$ comprises the following elements $H_{u}=\left\{x \in X_{u}: x u^{\prime}<x\right\}=\left\{x \in X: \tau(x)=u, x u^{\prime}<x\right\}$. Hence proving $H_{u} \subseteq \bigcup_{\kappa \ni s<{ }_{\kappa} u} \varsigma_{s \rightarrow u}\left(G_{s}\right)$ amounts to showing that in every densely ordered, odd or even involutive $\mathrm{FL}_{e}$-chain $\mathbf{X}=(X, \leq, \cdot, \rightarrow, t, f)$ with residual complement operation ', if $u \geq t$ and $u^{\prime}$ are idempotent, and $x \in X$ such that $\tau(x)=u$ and $x u^{\prime}<x$ then there exist a positive idempotent element $X \ni s<u$ and $y \in X$ such that $\tau(y)=s$ and $y u=x$. Let $x u^{\prime}<y<x$ (such $y$ exists since $X$ is densely ordered). Now, $y^{\prime}<\left(x u^{\prime}\right)^{\prime} \leq y^{\prime} u$ follows by 
claim (iv) in Lemma 3.1. Therefore, $u \notin S t a b_{y^{\prime}}$ and $s:=\tau(y)=\tau\left(y^{\prime}\right)<u$ follows. Since $y u \stackrel{(4.4)}{=} \rho_{s \rightarrow u}(y) \stackrel{(4.6)}{=} \rho_{u}(y) \stackrel{(4.18)}{=} \min \left\{z \in X_{u}: z \geq y\right\}$ and since $X_{u} \ni x u^{\prime}<y<x \in X_{u}$, to see that $y u=x$, it suffices to prove that $x u^{\prime}=x_{\downarrow_{u}}{ }^{19}$. But it holds true since $x \in H_{u}$ and $u^{\prime} \in H_{u}^{\bullet}$, and hence $x u^{\prime}=x u_{\downarrow_{u}}=x u^{\bullet} \stackrel{\text { Table }}{=}{ }^{\star \star(2,3)} x^{\bullet}=x_{\downarrow_{u}}$ follows from claim (iv) in the proof of Theorem $6.3 /(\mathrm{a})$, and we are done.

REMARK 8.4. The easiest way of generalizing Theorem 8.1 to conic algebras is to observe that there exist no conic odd or even involutive $\mathrm{FL}_{e}$-algebras which are not linearly ordered. Indeed, let $a, b \in X$. Proving that $a$ and $b$ are comparable, that is, $a \leq b$ or $b \leq a$ amounts to proving $a t \leq b$ or $b t \leq a$, or equivalently, $a \rightarrow b \geq t$ or $b \rightarrow a \geq t$ by adjointness. If $a \rightarrow b \geq t$ then $a \rightarrow b<t$ since $X$ is conic, hence $a \rightarrow b \leq f$ since $X$ is odd or even. By claims (i) and (ii) in Lemma 3.1, $a b^{\prime} \geq t$ follows. Therefore, $\left(a^{\prime} b\right)^{\prime} \geq t$ holds by claim (iii) in Lemma 3.1, hence $b \rightarrow a \geq t$ holds by claim (ii) in Lemma 3.1.

Acknowledgements. Open access funding provided by University of Pécs. The present scientific contribution was supported by the GINOP 2.3.2-152016-00022 grant and the Higher Education Institutional Excellence Programme 20765-3/2018/FEKUTSTRAT of the Ministry of Human Capacities in Hungary.

Open Access. This article is licensed under a Creative Commons Attribution 4.0 International License, which permits use, sharing, adaptation, distribution and reproduction in any medium or format, as long as you give appropriate credit to the original author(s) and the source, provide a link to the Creative Commons licence, and indicate if changes were made. The images or other third party material in this article are included in the article's Creative Commons licence, unless indicated otherwise in a credit line to the material. If material is not included in the article's Creative Commons licence and your intended use is not permitted by statutory regulation or exceeds the permitted use, you will need to obtain permission directly from the copyright holder. To view a copy of this licence, visit http://creativecommons.org/licenses/by/4.0/.

Publisher's Note Springer Nature remains neutral with regard to jurisdictional claims in published maps and institutional affiliations.

\section{References}

[1] Agliano, P., and F. Montagna,Varieties of BL-algebras I: general properties, Journal of Pure and Applied Algebra 181(2-3):105-129, 2003.

\footnotetext{
${ }^{19}$ Recall that $x_{\downarrow_{u}}$ is computed in $X_{u}$.
} 
[2] Anderson, A. R., and N.D. Belnap, Entailment. Volume I: The logic of relevance and necessity, Princeton University Press, Princeton, N. J., 1975.

[3] Anderson, M., and T. FeIL, Lattice-Ordered Groups: An Introduction, D. Reidel, 1988.

[4] Bahls, P., J. Cole, N. Galatos, P. Jipsen, and C., Tsinakis, Cancellative residuated lattices, Algebra Universalis, 50(1): 83-106, 2003.

[5] Baldi, P., A. Ciabattoni, and F. Gulisano, Standard completeness for extensions of IMTL, in 2017 IEEE International Conference on Fuzzy Systems (FUZZ-IEEE), 2017, pp. 1-6.

[6] Blok, W., and J. G. RAFtery, Varieties of commutative residuated integral pomonoids and their residuation subreducts, Journal of Algebra 190:280-328, 1997.

[7] Bonzio, S., Dualities for Plonka sums, Logica Universalis 12: 327-339, 2018.

[8] Bonzio, S., T. Moraschini, and M. Pra Baldi, Logics of left variable inclusion and Plonka sums of martices, Archive for Mathematical Logic 60:49-76, 2021.

[9] Cabrer, L. M., and S. A. Celani, Priestley dualities for some lattice-ordered algebraic structures, including MTL, IMTL and MV-algebras, Central European Journal of Mathematics 4(4): 600-623, 2006.

[10] Cabrer, L. M., and S. A. Celani, Kripke Semantics for Monoidal T-norm based Logics MTL and IMTL, CLE e-Prints 8(6), 2008; https://www.cle.unicamp.br/eprints/ index.php/CLE_e-Prints/article/view/914.

[11] Casari, E., Comparative logics and Abelian $\ell$-groups, in in R. Ferro, C. Bonotto, S. Valentini, and A. Zanardo, (eds.), Logic Colloquium '88, North Holland, Amsterdam, 1989, pp. 161-190.

[12] Cignoli, R. L. O., I. M. L. D’Ottaviano, and D. Mundici, Algebraic Foundations of Many-Valued Reasoning, vol. 7 of Trends in Logic, Kluwer Academic Publishers, 1999.

[13] Cignoli, R., and A. T. Torrell, Free Algebras in varieties of Glivenko MTLalgebras satisfying the equation $2\left(x^{2}\right)=(2 x)^{2}$, Studia Logica 83:157-181, 2006 .

[14] Cintula, P., P. HÁsek, and R. HorvčIK, Formal systems of fuzzy logic and their fragments, Annals of Pure and Applied Logic, 150(1-3):40-65, 2007.

[15] Dunn, J. M., Algebraic completeness results for R mingle and its extensions. Journal of Symbolic Logic 35(1):1-13, 1970.

[16] Esteva, F., J. Gispert, L. Godo and F. Montagna, On the standard and rational completeness of some axiomatic extensions of the Monoidal T-norm Logic, Studia Logica 71(2):199-226, 2002.

[17] Esteva, F., and L. Godo, Towards the generalization of Mundici's $\Gamma$ functor to IMTL algebras: The linearly ordered case, in: S. Aguzzoli, A. Ciabattoni, B. Gerla, C. Manara, and V. Marra, (eds.), Algebraic and Proof-theoretic Aspects of Non-classical Logics, vol. 4460 of Lecture Notes in Computer Science, Springer, Berlin, Heidelberg, 2007, pp. 127-137.

[18] Galatos, N., Minimal varieties of residuated lattices, AlgebraUniversalis 52(23):215-239, 2005.

[19] Galatos, N., P. Jipsen, T. Kowalski, and H. Ono, Residuated Lattices: An Algebraic Glimpse at Substructural Logics, Elsevier, Amsterdam, 2007. 
[20] Galatos, N., and J. G. RAftery, A category equivalence for odd Sugihara monoids and its applications, Journal of Pure and Applied Algebra 216: 2177-2192, 2012.

[21] Galatos, N., and J. G. Raftery, Idempotent residuated structures: Some category equivalences and their applications, Transactions of the American Mathematical Society 367:3189-3223,2015.

[22] Galatos, N., and C. Tsinakis, Generalized MV-algebras, Journal of Algebra 283:254-291, 2005.

[23] Gil-FÉrez, J., P. Jipsen and G. Metcalfe, Structure theorems for idempotent residuated lattices, Algebra Universalis, published online May 4, 2020, https://doi.org/10. 1007/s00012-020-00659-5

[24] Gispert, J., and A. Torrens, Axiomatic Extensions of IMT3 Logic, Studia Logica, 81(3):311-324, 2005.

[25] HÁJEK, P., Metamathematics of Fuzzy Logic, vol. 4 of Trends in Logic, Kluwer Academic Publishers, 1998.

[26] Horčık, R. Algebraic semantics, in: P. Cintula, P. Hájek, and C. Noguera, (eds.), Handbook of Mathematical Fuzzy Logic, College Publications, 2011, pp. 283-353.

[27] JENEI. S. Structure of left-continuous triangular norms with strong induced negations. (III) Construction and decomposition, Fuzzy Sets and Systems 128(2):197-208 2002.

[28] JEnei, S., Co-rotation, co-rotation-annihilation, and involutive ordinal sum constructions of residuated semigroups, in: Proceedings of the $19^{\text {th }}$ International Conference on Logic for Programming, Artificial Intelligence and Reasoning, Stellenbosch, South Africa, Springer, Heidelberg, 2013, pp.73

[29] JENEI, S., Group representation for even and odd involutive commutative residuated chains, arXiv:1910.01404, 2019.

[30] JENEI, S. Amalgamation and densification in classes of involutive commutative residuated lattices, arXiv:2012.14181, 2020.

[31] JeneI, S., The Hahn embedding theorem for a class of residuated semigroups, Studia Logica 108(6):1161-1206, 2020.

[32] Jenei, S., Correction to: The Hahn embedding theorem for a class of residuated semigroups, Studia Logica 109(4):887-901 2021.

[33] Jipsen, P., and F. Montagna, Embedding theorems for classes of GBL-algebras, Journal of Pure and Applied Algebra, 214:1559-1575, 2010.

[34] KüHR, J., Representable pseudo-BCK-algebras and integral residuated lattices, Journal of Algebra 317 (1):354-364, 2007.

[35] Lawson, M. V., Inverse Semigroups: The Theory of Partial Symmetries, World Scientific Publishing Company, 1998.

[36] Lawson, J., Fifty Years of Topological Algebra, Seminar Sophus Lie XXXIV, Workshop in honor of Karl Hofmann's 75th Birthday, Darmstadt, October 5-6, 2007.

[37] Liu, L. and X. Zhang, States on finite linearly ordered IMTL-algebras, Soft Computing 15:2021-2028, 2011.

[38] Metcalfe, G., Proof Theory for Propositional Fuzzy Logics, PhD thesis, King's College London 2003.

[39] Metcalfe, G., and F. Montagna, Substructural fuzzy logics, Journal of Symbolic Logic 72 (3):834-864, 2007. 
[40] Meyer, R. K., and J. K. Slaney, Abelian logic (from A to Z), in G. Presist, R. Routley and J. Norman, (eds.), Paraconsistent Logic: Essays on the Inconsistent, Philosophia, Munich, 1989, pp. 245-288.

[41] Montagna, F., and C. Tsinakis, Ordered groups with a conucleus, Journal of Pure and Applied Algebra 21481:71-88, 2010.

[42] Mostert, P. S., and A. L. Shields, On the structure of semigroups on a compact manifold with boundary, Annals of Mathematics 65:117-143, 1957.

[43] Mundici, D., Interpretation of $\mathrm{AF} \mathrm{C}^{*}$-algebras in Lukasiewicz sentential calculus, Journal of Functional Analysis 65 (1):15-63, 1986.

[44] Noguera, C., F. Esteva, and J. Gispert, On Some Varieties of MTL-algebras, Logic Journal of the IGPL 13(4):443-466, 2005.

[45] Noguera, C., F. Esteva, J. Gispert, Perfect and bipartite IMTL-algebras and disconnected rotations of prelinear semihoops, Archive for Mathematical Logic 44:869$886,2005$.

[46] Olson, J. S., Free representable idempotent commutative residuated lattices, International Journal of Algebra and Computation 18(8):1365-1394, 2008.

[47] Paoli, F., M. Spinks, and R. Verodd, Abelian Logic and the Logics of Pointed Lattice-Ordered Varieties, Logica Universalis 2(2):209-233, 2008.

[48] Plonka, J., On a method of construction of algebras, Fundamenta Mathematicae 61(2):183-189, 1967.

[49] Puhlmann, H., The snack powerdomain for database semantics, in: A. M. Borzyszkowski, and S. Sokolowski, (eds.,) Mathematical Foundations of Computer Science, Springer, Berlin, 1993, pp. 650-659

[50] Raftery, J. G., Representable idempotent commutative residuated lattices, Transactions of the American Mathematical Society 359:4405-4427, 2007.

[51] Saitô, T., Ordered inverse semigroups, Transactions of the American Mathematical Society 153:99-138, 1971.

[52] Wang, S., A Proof of the Standard Completeness for the Involutive Uninorm Logic, Symmetry 11:445, 2019.

\author{
S. JENEI \\ Institute of Mathematics and Informatics \\ University of Pécs \\ Ifjúság u. 6 \\ H-7624 Pécs \\ Hungary \\ jenei@ttk.pte.hu
}

